\title{
Noncommutative tori and the Riemann-Hilbert correspondence
}

\author{
Snigdhayan Mahanta and Walter D. van Suijlekom
}

\begin{abstract}
We study the interplay between noncommutative tori and noncommutative elliptic curves through a category of equivariant differential modules on $\mathbb{C}^{*}$. We functorially relate this category to the category of holomorphic vector bundles on noncommutative tori as introduced by Polishchuk and Schwarz and study the induced map between the corresponding K-theories. In addition, there is a forgetful functor to the category of noncommutative elliptic curves of Soibelman and Vologodsky, as well as the forgetful functor to the category of vector bundles on $\mathbb{C}^{*}$ with regular singular connections.

The category that we consider has the nice property of being a Tannakian category, hence it is equivalent to the category of representations of an affine group scheme. Via an equivariant version of the Riemann-Hilbert correspondence we determine this group scheme to be (the algebraic hull of) $\mathbb{Z}^{2}$. We also obtain a full subcategory of the holomorphic vector bundles on the noncommutative torus which is equivalent to the category of representations of $\mathbb{Z}$. This group is the proposed topological fundamental group of the noncommutative torus (understood as a degenerate elliptic curve) and we study Nori's notion of étale fundamental group in this context.
\end{abstract}

Mathematics Subject Classification (2000). 34M50, 58B34; 14A22, 32L05.

Keywords. Noncommutative tori, fundamental group, semistable bundles.

\section{Introduction}

Noncommutative geometry in its various forms has come to the forefront of mathematical research lately and noncommutative tori constitute perhaps the most extensively studied class of examples of noncommutative differentiable manifolds. They were introduced by Connes during the early 1980s [3] and were systematically studied by Connes [3], Rieffel [27], [28] and others. Recently Polishchuk and Schwarz have provided a new perspective on them which is quite amenable to techniques in algebraic geometry [23], [21]. At the same time Soibelman and Vologodsky have introduced noncommutative elliptic curves as certain equivariant categories of coherent sheaves [31]. The guiding principle behind both constructions is replacing a mathematical object by its category of appropriately defined representations, viz., vector bundles 
with connections in the former case, denoted by $\operatorname{Vect}\left(\mathbb{T}_{\theta}^{\tau}\right)$, and coherent sheaves in the latter, denoted by $\mathscr{B}_{q}$, where $q=e^{2 \pi i \theta}$ and $\theta$ is an irrational number.

In this article we try to connect the above two constructions by introducing an intermediate category $\mathscr{B}_{q}^{\tau}$. Besides the existence of a forgetful functor from $\mathscr{B}_{q}^{\tau}$ to $\mathscr{B}_{q}$ (as the notation might suggest), we construct a faithful and exact functor from $\mathscr{B}_{q}^{\tau}$ to $\operatorname{Vect}\left(\mathbb{T}_{\theta}^{\tau}\right)$. One may view $\mathscr{B}_{q}^{\tau}$ as a sort of categorical 'correspondence' between $\operatorname{Vect}\left(\mathbb{T}_{\theta}^{\tau}\right)$ and $\mathscr{B}_{q}$. The category $\mathscr{B}_{q}^{\tau}$ turns out to be a Tannakian category. Via an equivariant version of the Riemann-Hilbert correspondence we show that it is equivalent to the category of finite dimensional representations of (the algebraic hull of) $\mathbb{Z}^{2}$ (see Theorem 19). This allows us to describe the K-theory of $\mathscr{B}_{q}^{\tau}$ as the free abelian group generated by two copies of $\mathbb{C}^{*}$ (see Corollary 21 ).

The final part of the paper is a little speculative in nature, in which we propose a fundamental group for the noncommutative torus, both topological and étale. For the latter, Nori's approach [16], [17] to étale fundamental groups of smooth quasiprojective curves involving Tannakian categories plays a central motivating role.

This paper is organized as follows. In the first section we briefly review the main results of [23], including the basic definitions and examples. We also discuss the rudiments of noncommutative tori, which are relevant for our purposes as it is known that there are several ways of looking at them. We also show that there is a certain modularity property satisfied by the categories $\operatorname{Vect}\left(\mathbb{T}_{\theta}^{\tau}\right)$ (see Proposition 1).

In the second section we first provide a motivation for the definition of the categories $\mathscr{B}_{q}^{\tau}$ and then construct a faithful and exact functor from $\mathcal{B}_{q}^{\tau}$ into $\operatorname{Vect}\left(\mathbb{T}_{\theta}^{\tau}\right)$. We also give a description of the image of our functor and discuss the induced map on the K-theories of the corresponding categories.

In the third section we start by briefly recalling some preliminaries of Tannakian categories. We explain the structure of a Tannakian category on the category $\mathscr{B}_{q}^{\tau}$ and prove an equivariant version of the Riemann-Hilbert correspondence on $\mathbb{C}^{*}$. Via this correspondence, we find that $\mathscr{B}_{q}^{\tau}$ is equivalent to the category of finite dimensional representations of $\mathbb{Z}^{2}$. As a consequence we are able to compute the K-theory of $\mathfrak{B}_{q}^{\tau}$.

We conclude with the proposal for the fundamental group of the noncommutative torus as alluded to before. After a short discussion on Nori finite bundles involving stability conditions, we establish a full subcategory $\mathscr{B}^{\tau}$ of $\operatorname{Vect}\left(\mathbb{T}_{\theta}^{\tau}\right)$ which is a Tannakian category, and equivalent to $\operatorname{Rep}(\mathbb{Z})$. We define a Tannakian subcategory of $\mathscr{B}^{\tau}$, whose associated group scheme is our proposal for the étale fundamental group of the noncommutative torus.

Convention. In this article, unless otherwise stated, $\theta$ is always assumed to be irrational and $\tau$ in the lower half plane as in [23]. The ground field is also assumed to be $\mathbb{C}$.

Acknowledgements. Both authors would like to thank Matilde Marcolli for intensive discussions, as well as Masoud Khalkhali, Emanuele Macrí and Jorge Plazas for 
helpful comments. The first author would also like to thank Vasilisa Shramchenko for indicating the reference [32] and Christian Kaiser, E. Lee Lady and Fernando Muro for several useful discussions. The second author would like to thank Eli Hawkins, Michael Müger and Marius van der Put for helpful comments. Both authors would also like to thank the Max-Planck-Institut für Mathematik, Bonn for hospitality and support. The first author also acknowledges the hospitality of the Fields Institute, Toronto, and the second author the Hausdorff Research Institute for Mathematics, Bonn, where a part of this work was carried out.

\section{Preliminaries}

We recall some basic facts about the structure of the category of holomorphic bundles over noncommutative tori.

1.1. Holomorphic bundles on noncommutative tori. The noncommutative torus is a particular case of a transformation group $C^{*}$-algebra, with $\mathbb{Z}$ acting continuously on the $C^{*}$-algebra $C\left(\mathbb{\$}^{1}\right)$ of continuous functions on the circle. Pimsner and Voiculescu [18] and separately Rieffel [27] studied their K-theory, while Connes analysed their differential structure [3]. We will work with the smooth noncommutative torus, which is a dense Fréchet subalgebra of this transformation group $C^{*}$-algebra.

Let $\theta$ be an irrational real number. The algebra of smooth functions $\mathcal{A}_{\theta}$ on the noncommutative torus $\mathbb{T}_{\theta}$ consists of elements of the form $\sum_{\left(n_{1}, n_{2}\right) \in \mathbb{Z}^{2}} a_{n_{1}, n_{2}} U_{1}^{n_{1}} U_{2}^{n_{2}}$ with $\left(n_{1}, n_{2}\right) \rightarrow a_{n_{1}, n_{2}}$ rapidly decreasing and $U_{1}, U_{2}$ are unitaries satisfying the commutation relation

$$
U_{2} U_{1}=\exp (2 \pi i \theta) U_{1} U_{2} .
$$

A less ad hoc definition of $\mathcal{A}_{\theta}$ is given as the smooth crossed product $C^{\infty}\left(\mathbb{S}^{1}\right) \rtimes_{\theta} \mathbb{Z}$ with $\mathbb{Z}$ acting on $\mathbb{S}^{1}$ by rotation over $\theta$; this is the smooth analogue of the aforementioned transformation group $C^{*}$-algebra. The Fourier transform then establishes the isomorphism $\mathscr{A}_{\theta} \simeq C^{\infty}\left(\Phi^{1}\right) \rtimes_{\theta} \mathbb{Z}$.

The two basic derivations $\delta_{1}$ and $\delta_{2}$ acting on $\mathcal{A}_{\theta}$ are as follows:

$$
\delta_{j}\left(\sum_{\left(n_{1}, n_{2}\right) \in \mathbb{Z}^{2}} a_{n_{1}, n_{2}} U_{1}^{n_{1}} U_{2}^{n_{2}}\right)=2 \pi i \sum_{\left(n_{1}, n_{2}\right) \in \mathbb{Z}^{2}} n_{j} a_{n_{1}, n_{2}} U_{1}^{n_{1}} U_{2}^{n_{2}} \quad(j=1,2) .
$$

Equivalently, one can define $\delta_{1}$ and $\delta_{2}$ by $\delta_{j}\left(U_{i}\right)=2 \pi i \delta_{i j} U_{i}$ which is then extended to the whole of $\mathcal{A}_{\theta}$ by applying the Leibniz rule.

The derivations $\delta_{1}$ and $\delta_{2}$ are the infinitesimal generators of the action of a commutative torus $\mathbb{T}^{2}$ on $\mathcal{A}_{\theta}$ by automorphisms. Inside the complexified Lie algebra generated by $\delta_{1}$ and $\delta_{2}$, we are interested in the vector parametrized by two complex numbers $\omega_{1}$ and $\omega_{2}$. We denote

$$
\delta_{\omega}=\omega_{1} \delta_{1}+\omega_{2} \delta_{2}
$$


If $\omega=(\tau, 1)$ we also set $\delta_{\tau}=\delta_{\omega}$, which is the so-called complex structure on $\mathcal{A}_{\theta}$ as in [5].

1.1.1. The category of holomorphic bundles on $\mathbb{T}_{\boldsymbol{\theta}}$. The Serre-Swan Theorem establishes an equivalence between the category of vector bundles over a topological space $M$ and finitely generated projective modules (henceforth, for brevity, referred to as finite projective modules) over $C(M)$. In this spirit, it makes sense to define vector bundles over the noncommutative torus $\mathbb{T}_{\theta}$ as finite projective right $\mathcal{A}_{\theta}$-modules.

In [3], Connes has constructed finite projective modules over $\mathcal{A}_{\theta}$ that are labelled by a tuple $(m, n) \in \mathbb{Z}^{2}$. Later, in [27] Rieffel has shown that this set, in fact, exhausts the complete set of finite projective modules over $\mathcal{A}_{\theta}$ (up to isomorphism).

We generalize the category considered by Polishchuk and Schwarz slightly by defining the objects of the category $\operatorname{Vect}\left(\mathbb{T}_{\theta}^{\omega}\right)$ to be finite projective right $\mathcal{A}_{\theta}$-modules carrying a holomorphic structure which is a lifting of $\delta_{\omega}$. More precisely, a holomorphic structure on a finite projective $\mathcal{A}_{\theta}$-module $E$ is given by a $\mathbb{C}$-linear connection $\nabla: E \rightarrow E$ satisfying the Leibniz rule

$$
\nabla(e a)=\nabla(e) a+e \delta_{\omega}(a) \quad\left(\text { for all } e \in E, a \in \mathcal{A}_{\theta}\right) .
$$

A morphism $h: E \rightarrow E^{\prime}$ is said to be holomorphic if it commutes with the connection, i.e., $\nabla_{E^{\prime}}(h e)=h \nabla_{E}(e)$. These are the morphisms of the category.

One defines the cohomology groups $H^{0}$ (resp. $H^{1}$ ) of $\mathcal{A}_{\theta}$ with respect to a holomorphic bundle $E$, equipped with a connection $\nabla$, as the kernel (resp. cokernel) of $\nabla$.

If $\omega=(\tau, 1)$, then $\operatorname{Vect}\left(\mathbb{T}_{\theta}^{\omega}\right)$ reduces to the category of holomorphic bundles $\operatorname{Vect}\left(\mathbb{T}_{\theta}^{\tau}\right)$ as introduced in [23].

Proposition 1. (a) If $g$ is an element in $\operatorname{SL}(2, \mathbb{Z})$, then $\operatorname{Vect}\left(\mathbb{T}_{\theta}^{g \omega}\right) \simeq \operatorname{Vect}\left(\mathbb{T}_{\theta}^{\omega}\right)$.

(b) If $\omega_{2} \neq 0$ and $\tau=\frac{\omega_{1}}{\omega_{2}}$, then $\operatorname{Vect}\left(\mathbb{T}_{\theta}^{\omega}\right) \simeq \operatorname{Vect}\left(\mathbb{T}_{\theta}^{\tau}\right)$.

Proof. (a) Given a $g \in \operatorname{SL}(2, \mathbb{Z})$, we construct a $*$-automorphism $\sigma$ of $\mathcal{A}_{\theta}$ such that $\sigma^{-1} \delta_{\omega} \sigma=\delta_{g \omega}$. Evidently, it is enough to do this for the generators of $\operatorname{SL}(2, \mathbb{Z})$, i.e., $g_{1}=\left(\begin{array}{ll}1 & 1 \\ 0 & 1\end{array}\right)$ and $g_{2}=\left(\begin{array}{cc}0 & -1 \\ 1 & 0\end{array}\right)$. For $g_{1}, \delta_{g_{1} \omega}=\left(\omega_{1}+\omega_{2}\right) \delta_{1}+\delta_{2}$. We define $\sigma_{1}: \mathcal{A}_{\theta} \rightarrow \mathcal{A}_{\theta}$ as $\sigma_{1}\left(U_{1}\right)=U_{1} U_{2}, \sigma_{1}\left(U_{2}\right)=U_{2}$. One may easily check that $\sigma_{1}\left(U_{1}\right)$ and $\sigma_{1}\left(U_{2}\right)$ satisfy the commutation relation of $\mathcal{A}_{\theta}$ as in eqn. (1) and also that

$$
\sigma_{1}^{-1} \delta_{\omega} \sigma_{1}\left(U_{1}\right)=\delta_{g_{1} \omega}\left(U_{1}\right)
$$

Similarly, for $U_{2}$ one may check that the actions of $\delta_{\omega}$ and $\delta_{g_{1} \omega}$ agree. For $g_{2}$, $\delta_{g_{2} \omega}=-\omega_{2} \delta_{1}+\omega_{1} \delta_{2}$ and we define $\sigma_{2}\left(U_{1}\right)=U_{2}^{-1}, \sigma_{2}\left(U_{2}\right)=U_{1}$. Once again one can easily check that the new generators satisfy eqn. (1) and that the actions of $\delta_{\omega}$ and $\delta_{g_{2} \omega}$ agree on $U_{1}$ and $U_{2}$. Explicitly, the functor sends $\left(\mathcal{A}_{\theta}, \delta_{\omega}\right)$ to $\left(\mathcal{A}_{\theta}, \delta_{g_{i} \omega}\right)$, $i=1,2$, and twists the module structure by $\sigma_{i}, i=1$, 2, i.e., $e \cdot a:=e \sigma_{i}(a), i=1,2$ 
and $e \in E$. One verifies that $\nabla$ on $E$ is compatible with $\delta_{g_{i} \omega}, i=1$, 2, with respect to the twisted module structure. Indeed,

$$
\nabla(e \cdot a)=\nabla\left(e \sigma_{i}(a)\right)=\nabla(e) \cdot a+e \cdot \delta_{g_{i} \omega}(a)
$$

where $e \in E, a \in \mathcal{A}_{\theta}$ and $i=1,2$.

(b) In our notation, $\delta_{\tau}=\frac{\delta_{\omega}}{\omega_{2}}$. Sending each $\nabla$ to $\nabla^{\prime}:=\frac{\nabla}{\omega_{2}}$ makes $\nabla^{\prime}$ automatically compatible with $\delta_{\tau}$. More precisely, the functor sends $\left(\mathcal{A}_{\theta}, \delta_{\omega}\right)$ to $\left(\mathcal{A}_{\theta}, \delta_{\tau}\right)$ and $(E, \nabla)$ to $\left(E, \nabla^{\prime}\right)$.

We end this section with a technical result that we will need later on. Let us define $\operatorname{wd}(\tau):=\bar{\tau} \tau /|\operatorname{Re}(\tau)|$ as the 'real width' of a transversal to $\tau \mathbb{Z}$ or, equivalently, the (interval) length of the intersection of a transversal to $\tau \mathbb{Z}$ with the reals $\mathbb{R} \subset \mathbb{C}$. Before we explain how this can be achieved, recall that a transversal to $\tau \mathbb{Z}$ in $\mathbb{C}$ is the image of a section of the projection map $\mathbb{C} \rightarrow \mathbb{C} / \tau \mathbb{Z}$ (e.g., the strip $0 \leq \operatorname{Re}(z / \tau)<1$ ).

Lemma 2. For any $\tau \in \mathbb{C}$ there is an element $g$ in $\operatorname{SL}(2, \mathbb{Z})$ such that $\operatorname{wd}(g \tau)<1$.

Proof. One simply computes that for $-(\tau+N)^{-1}$ (obtained as translation by an integer $N$ composed with inversion) we have

$$
\operatorname{wd}\left(-(\tau+N)^{-1}\right)=\frac{1}{\operatorname{Re}(\tau)+N} .
$$

Thus, for any $\tau$ there exists an integer $N>0$ such that $\operatorname{wd}\left(-(\tau+N)^{-1}\right)<1$.

1.1.2. The derived category. The derived category of holomorphic bundles is $d e$ fined as the homotopy category of a DG category (or a differential graded category), which is a category with the Hom sets carrying a structure of a differential graded complex of $\mathbb{C}$-vector spaces (see [11] for more details). The corresponding homotopy category is obtained by replacing the Hom complexes by their zeroth cohomologies. Henceforth, by $\mathbb{T}_{\theta}^{\tau}$ we shall mean the noncommutative torus $\mathbb{T}_{\theta}$ equipped with the derivation $\delta_{\tau}$. The DG category in consideration, denoted by $\mathcal{C}(\theta, \tau)$, consists of objects of $\operatorname{Vect}\left(\mathbb{T}_{\theta}^{\tau}\right)$, labelled by an integer indicating its translation degree. The Hom's in $\mathcal{C}(\theta, \tau)$ are given by a differential complex made up from the connections on the Hom sets in $\operatorname{Vect}\left(\mathbb{T}_{\theta}^{\tau}\right)$. Note that the Hom sets in $\operatorname{Vect}\left(\mathbb{T}_{\theta}^{\tau}\right)$ also carry a module structure over some noncommutative torus (not necessarily $\mathbb{T}_{\theta}$ ).

Polishchuk and Schwarz constructed a functor from the DG category $\ell(\theta, \tau)$ to $D^{b}\left(X_{\tau}\right)$ and showed that the induced functor on the cohomology category is fully faithful and that the image of $\operatorname{Vect}\left(\mathbb{T}_{\theta}^{\tau}\right)$ lies in the heart of the $t$-structure 
$\left(D^{\theta, \leqslant 0}, D^{\theta, \geqslant}\right)$, where

$$
\begin{aligned}
D^{\theta, \leqslant 0}=\left\{K^{\bullet} \in D^{b}\left(X_{\tau}\right) \mid H^{>0}\left(K^{\bullet}\right)=0\right. & \\
& \text { all ss factors of } \left.H^{0}\left(K^{\bullet}\right) \text { have slope }>\theta\right\} \\
D^{\theta, \geqslant 0}=\left\{K^{\bullet} \in D^{b}\left(X_{\tau}\right) \mid H^{<-1}\left(K^{\bullet}\right)=0,\right. & \\
& \text { all ss factors of } \left.H^{-1}\left(K^{\bullet}\right) \text { have slope } \leqslant \theta\right\},
\end{aligned}
$$

with ss denoting semistable (see for the definition of slope Section 1.2 below).

Then Polishchuk showed that this functor actually induces an equivalence between $\operatorname{Vect}\left(\mathbb{\nabla}_{\theta}^{\tau}\right)$ and the heart $\mathcal{C}^{\theta, \tau}$ of the above $t$-structure [20], whose derived category is again equivalent to $D^{b}\left(X_{\tau}\right)$ [21]. This implies that $\operatorname{Vect}\left(\mathbb{T}_{\theta}^{\tau}\right)$ is abelian and its derived category is equivalent to $D^{b}\left(X_{\tau}\right)$ via the Polishchuk-Schwarz functor, denoted by s: $H^{0} \mathcal{C}(\theta, \tau) \rightarrow D^{b}\left(X_{\tau}\right)$.

Remark 3. The functor $\delta_{\tau}$ actually induces an equivalence between $\operatorname{Vect}\left(\mathbb{T}_{\theta}^{\tau}\right)$ and $\mathcal{C}^{-\theta^{-1}, \tau}$. Observe that $\left(\begin{array}{cc}0 & 1 \\ -1 & 0\end{array}\right) \theta=-\theta^{-1}$ says that $A_{-\theta^{-1}}$ is Morita equivalent to $\mathcal{A}_{\theta}$.

Summarising, one has the following equivalences of categories

$$
\operatorname{Vect}\left(\mathbb{\mathbb { T }}_{\theta}^{\tau}\right) \cong \operatorname{Vect}\left(\mathbb{\mathbb { T }}_{-\theta^{-1}}^{\tau}\right) \cong \ell^{\theta, \tau} \text { and } D^{b}\left(\iota^{\theta, \tau}\right) \cong D^{b}\left(X_{\tau}\right)
$$

1.2. Standard bundles over $\mathbb{T}_{\boldsymbol{\theta}}^{\boldsymbol{\tau}}$. In $\operatorname{Vect}\left(\mathbb{T}_{\theta}^{\tau}\right)$ there are certain distinguished objects called standard holomorphic bundles whose images under the Polishchuk-Schwarz functor $\delta_{\tau}$ are stable objects inside $D^{b}\left(X_{\tau}\right)$, i.e., objects of the form $\mathscr{F}[n]$ where $\mathcal{F}$ is a stable bundle on $X_{\tau}$ or the structure sheaf of a point [23]. The underlying $\mathcal{A}_{\theta}$-module of a standard holomorphic bundle is of the form $E_{m, n}$ with $m, n$ coprime as constructed in [3]. One defines its degree and rank as

$$
\operatorname{deg}\left(E_{m, n}\right):=m, \quad \operatorname{rk}\left(E_{m, n}\right):=m \theta+n,
$$

whereas the slope of the bundle is defined as $\mu\left(E_{m, n}\right):=\frac{m}{m \theta+n}$.

If $m \neq 0$, given any $z \in \mathbb{C}$ one endows $E_{m, n}$ with a connection of the form

$$
\nabla_{z}(f)=\frac{\partial f}{\partial x}+2 \pi i\left(\tau \mu\left(E_{m, n}\right) x+z\right) f .
$$

If $m=0$, for a given $z \in \mathbb{C}$ one equips $E_{0,1}=\mathcal{A}_{\theta}$ with the connection $\nabla_{z}(a)=$ $\delta_{\tau}(a)+2 \pi i z \cdot a$. A standard holomorphic bundle is a module $E_{m, n}$ with $m \theta+n>0$ and equipped with this special connection $\nabla_{z}$. They are labelled by two coprime integers and a complex parameter. We set $E_{m, n}^{z}=\left(E_{m, n}, \nabla_{z}\right)$; the modules $E_{0,1}^{z}=$ $\left(\mathcal{A}_{\theta}, \nabla_{z}\right)$ will be abbreviated to $E_{1}^{z}$. 


\section{Equivariant coherent sheaves and $\operatorname{Vect}\left(\mathbb{T}_{\theta}^{\tau}\right)$}

We observe that there is an honest action of $\theta \mathbb{Z}$ on $X_{\tau}$ and hence on $\operatorname{Coh}\left(X_{\tau}\right)$. Indeed, the point $\theta \bmod (\mathbb{Z}+\tau \mathbb{Z})$ on $X_{\tau}$ lies on the real axis of the fundamental domain of the torus and its action is restricted to the circle obtained by folding this axis. In fact, the action given by translations of $\theta$ on $X_{\tau}$ transforms to the action of multiplication by powers of $q=e^{2 \pi i \theta}$ under the Jacobi uniformization, i.e., $z \mapsto q z$ on $\mathbb{C}^{*} / \tilde{q}^{\mathbb{Z}}$, $\tilde{q}=e^{2 \pi i \tau}$. So we are confronted with a double quotient problem, where the actions commute. Namely, it is the improper action of the group $q^{\mathbb{Z}}$ on $X_{\tau}$, which is itself obtained by the free and proper action of the group $\tilde{q}^{\mathbb{Z}}$ on $\mathbb{C}^{*}$ (both actions are by multiplication). Soibelman and Vologodsky have described the quotient space $\mathbb{C}^{*} / q^{\mathbb{Z}}$ in terms of their noncommutative elliptic curves $\mathscr{B}_{q}$ in [31]. The category $\mathscr{B}_{q}$ is nothing but the category of $q^{\mathbb{Z}}$-equivariant (analytic) coherent sheaves on $\mathbb{C}^{*}$ (or equivalently, the category of modules over the crossed product algebra $\mathcal{O}\left(\mathbb{C}^{*}\right) \rtimes_{q} \mathbb{Z}$, which are finitely presentable over $\mathcal{O}\left(\mathbb{C}^{*}\right)$ ). It follows from Lemma 3.2 of [31] that for any $M \in \mathscr{B}_{q}$ the underlying $\mathcal{O}\left(\mathbb{C}^{*}\right)$-module is free. However, there are interesting actions of $\theta \mathbb{Z}$ or $q^{\mathbb{Z}}$ on the free modules with respect to which they are equivariant. Let us denote by $\alpha$ the induced action by automorphisms of $\theta \mathbb{Z}$ on $\mathcal{O}\left(\mathbb{C}^{*}\right)$ :

$$
\alpha(f)(z)=f(q z) \quad\left(z \in \mathbb{C}^{*}, q=e^{2 \pi i \theta}\right) .
$$

Here we have understood the notation $\alpha:=\alpha(1)$ for the generator of $\mathbb{Z}$, so that $\alpha(n)=\alpha^{n}$. What is lacking in this picture is an infinitesimal action in terms of $\delta_{\tau}$ and compatible connections, which accounts for the remaining $\tau \mathbb{Z}$ quotient operation. To this end, we define a derivation on $\mathcal{O}\left(\mathbb{C}^{*}\right)$ by $\delta=\tau z \frac{d}{d z}$. It is this infinitesimal action by $\delta$ that will turn out to be the appropriate replacement for the infinitesimal action of the group $\tau \mathbb{Z}$.

2.1. The category $\mathscr{B}_{\boldsymbol{q}}^{\boldsymbol{\tau}}$. Our goal in this section is to define a category alluded to before, which is somehow 'in between' the categories $\operatorname{Vect}\left(\mathbb{T}_{\theta}^{\tau}\right)$ introduced by Polishchuk and Schwarz and $\mathscr{B}_{q}$ by Soibelman and Vologodsky. More precisely, we would like to construct a category $\mathscr{B}_{q}^{\tau}$ that is functorially related to both of these categories. At the same time, we would like to stay as close as possible to the setting of the Riemann-Hilbert correspondence. The discussion above motivates us to define the following category as a description of the quotient of $\mathscr{B}_{q}$ by the infinitesimal action of $\tau \mathbb{Z}$.

Definition 4. The category $\mathscr{B}_{q}^{\tau}$ consists of triples $(M, \sigma, \nabla)$, where

- $M$ is a finitely presentable $\mathcal{O}\left(\mathbb{C}^{*}\right)$-module, i.e., there is an exact sequence,

$$
\mathcal{O}\left(\mathbb{C}^{*}\right)^{m} \rightarrow \mathcal{O}\left(\mathbb{C}^{*}\right)^{n} \rightarrow M \rightarrow 0
$$


- $\sigma$ is a representation of $\theta \mathbb{Z}$ on $M$ covering the action $\alpha$ of $\theta \mathbb{Z}$ on $\mathcal{O}\left(\mathbb{C}^{*}\right)$, i.e.,

$$
\sigma(m \cdot f)=\sigma(m) \cdot \alpha(f) \quad\left(m \in M, f \in \mathcal{O}\left(\mathbb{C}^{*}\right)\right) ;
$$

- $\nabla$ is a $\theta \mathbb{Z}$-equivariant connection on $M$ covering the derivation $\delta=\tau z \frac{d}{d z}$ on $\mathcal{O}\left(\mathbb{C}^{*}\right)$, i.e., it satisfies

$$
\begin{aligned}
& \nabla(m \cdot f)=\nabla(m) \cdot f+m \cdot \delta(f), \\
& \nabla(\sigma(m))=\sigma(\nabla(m))
\end{aligned}
$$

for all $m \in M, f \in \mathcal{O}\left(\mathbb{C}^{*}\right)$.

In addition, we impose that the connection $\nabla$ is a regular singular connection on $M$, that is, there exists a module basis $\left\{e_{1}, \ldots, e_{n}\right\}$ of $M$ for which the holomorphic functions (on $\left.\mathbb{C}^{*}\right) z^{-1} A_{i j}(i, j=1, \ldots, n)$ defined by $A_{i j} e_{j}=\nabla\left(e_{i}\right)$ have simple poles at 0 . We call $A=\left(A_{i j}\right)$ the matrix of the connection with respect to that module basis.

The morphisms in this category are equivariant $\mathcal{O}\left(\mathbb{C}^{*}\right)$-module maps that are compatible with the connections. We will also write $M=(M, \sigma, \nabla)$ when no confusion can arise. For two objects $M$ and $N$ we denote by $\operatorname{Hom}_{\mathcal{O}\left(\mathbb{C}^{*}\right)}^{\theta \mathbb{Z}, \delta}(M, N)$ the $\mathbb{C}$-linear vector space of morphisms between them.

The uniqueness of the matrix $A=A_{i j}$ (after the choice of a module basis $\left\{e_{i}\right\}$ for $M$ ) is due to the fact that the modules $M$ in $\mathscr{B}_{q}^{\tau}$ turn out to be free as $\mathcal{O}\left(\mathbb{C}^{*}\right)$-modules. This was observed in [31], Lemma 2, and used the fact that the sheaf $M \otimes_{\mathbb{C}} \mathcal{O}_{\mathbb{C}^{*}}$ must be torsion-free due to $\theta \mathbb{Z}$-equivariance. Hence it is locally free on $\mathbb{C}^{*}$ and thus a trivial vector bundle. It also follows from the fact that a coherent sheaf admitting a connection is automatically locally free. As a consequence the $\mathcal{O}\left(\mathbb{C}^{*}\right)$-module of its global sections is free. This freeness as $\mathcal{O}\left(\mathbb{C}^{*}\right)$-modules can be translated into freeness as $\theta \mathbb{Z}$-equivariant $\mathcal{O}\left(\mathbb{C}^{*}\right)$-modules as follows. Suppose that $M \simeq V \otimes_{\mathbb{C}} \mathcal{O}\left(\mathbb{C}^{*}\right)$ as $\mathcal{O}\left(\mathbb{C}^{*}\right)$-modules with $V$ a complex vector space. Via this identification there is an induced action of $\theta \mathbb{Z}$ on $V \otimes \mathbb{C} \mathcal{O}\left(\mathbb{C}^{*}\right)$ making this an isomorphism of $\theta \mathbb{Z}$-equivariant $\mathcal{O}\left(\mathbb{C}^{*}\right)$-modules.

Remark 5. The $\tau$-dependence in the above construction might seem artificial, since all categories $\mathscr{B}_{q}^{\tau}$ are equivalent for all $\tau$. Our notation is motivated by the relation of $\mathscr{B}_{q}^{\tau}$ with $\operatorname{Vect}\left(\mathbb{T}_{\theta}^{\tau}\right)$ which is established below. An equivalent construction could be obtained by translating the $\tau$-dependence from the category $\mathscr{B}_{q}^{\tau}$ to the functor to $\psi^{*}: \mathscr{B}_{q}^{\tau} \rightarrow \operatorname{Vect}\left(\mathbb{T}_{\theta}^{\tau}\right)$ of Proposition 8 below.

Let $\mathcal{B}^{\tau}$ denote the category of pairs $(V, \nabla)$ with $V$ a vector bundle on $\mathbb{C}^{*}$ and $\nabla$ a regular singular connection on $V$ associated to $\delta=\tau z \frac{d}{d z}$. By the above remarks on the modules $M$ in $\mathscr{B}_{q}^{\tau}$, there is a functor from $\mathcal{B}_{q}^{\tau}$ to $\mathscr{B}^{\tau}$ which forgets the action 
of $\theta \mathbb{Z}$. Due to Deligne [6] (see also, for instance, Theorem 1.1 and the paragraph after Remark 1.2 of [14]), we know that the category $\mathcal{B}^{\tau}$ is equivalent to the category of finite dimensional representations of the fundamental group $\pi_{1}\left(\mathbb{C}^{*}, z^{\prime}\right) \simeq \mathbb{Z}$ with a base point $z^{\prime}$. This result motivates the regular singularity condition we have imposed on the connections in Definition 4.

In Section 3 we will enhance this Riemann-Hilbert correspondence to an equivariant version and show that a similar statement holds for $\mathcal{B}_{q}^{\tau}$. Let us first proceed to examine some of the properties of $\mathscr{B}_{q}^{\tau}$ and its relation with the other two categories, viz., $\mathscr{B}_{q}$ and $\operatorname{Vect}\left(\mathbb{T}_{\theta}^{\tau}\right)$.

Proposition 6. The category $\mathscr{B}_{q}^{\tau}$ is an abelian category.

Proof. It is proven in Proposition 3.3 of [31] that the category $\mathscr{B}_{q}$ is abelian. One observes readily that there is a faithful functor (forgetting the connection) from $\mathscr{B}_{q}^{\tau}$ to $\mathscr{B}_{q}$. Suppose that $f: M \rightarrow N$ is a morphism in $\mathscr{B}_{q}^{\tau}$. Since it is also a morphism in $\mathscr{B}_{q}$, both $\operatorname{ker} f$ and coker $f$ are equivariant $\mathcal{O}\left(\mathbb{C}^{*}\right)$-modules. Moreover, the map $f$ intertwines the connections on $M$ and $N$ and hence induces compatible connections on ker $f$ and coker $f$ making them objects in $\mathscr{B}_{q}^{\tau}$.

We now view $\mathcal{A}_{\theta}$ as a module over $\mathcal{O}\left(\mathbb{C}^{*}\right)$ via the homomorphism

$$
\psi: \mathcal{O}\left(\mathbb{C}^{*}\right) \rightarrow \mathcal{A}_{\theta}, \quad \sum_{n \in \mathbb{Z}} f_{n} z^{n} \mapsto \sum_{n \in \mathbb{Z}} f_{n} U_{1}^{n} .
$$

This is well defined since a sequence $f_{n}$ of exponential decay is certainly a Schwartz sequence.

Remark 7. The map is essentially restricting a holomorphic function on $\mathbb{C}^{*}$ to the unit circle. In fact, it is injective since, if a holomorphic function vanishes on the unit circle, it must vanish on the whole of $\mathbb{C}^{*}$. Note that $\mathscr{A}_{\theta}$ is not finitely generated over $\mathcal{O}\left(\mathbb{C}^{*}\right)$ and hence not an element of $\mathscr{B}_{q}$ or $\mathscr{B}_{q}^{\tau}$.

Proposition 8. The following association defines a right exact functor, denoted $\psi_{*}$, from $\mathscr{B}_{q}^{\tau}$ to $\operatorname{Vect}\left(\mathbb{\nabla}_{\theta}^{\tau}\right)$. For an object $(M, \sigma, \nabla)$ in $\mathcal{B}_{q}^{\tau}$ we define an object $(\tilde{M}, \widetilde{\nabla})$ in $\operatorname{Vect}\left(\mathbb{T}_{\theta}^{\tau}\right)$ by

$$
\tilde{M}=M \otimes{\mathcal{O}\left(\mathbb{C}^{*}\right)}_{\mathcal{A}_{\theta}} \tilde{\nabla}=2 \pi i \nabla \otimes 1+1 \otimes\left(\tau \delta_{1}+\delta_{2}\right)=2 \pi i \nabla \otimes 1+1 \otimes \delta_{\tau} .
$$

Proof. Observe that $\psi(2 \pi i \delta f)=\tau \delta_{1}(\psi(f))$ as follows from the definitions of $\delta$ and $\delta_{1}$. Moreover, the image of $\mathcal{O}\left(\mathbb{C}^{*}\right)$ under the map $\psi$ lies in the kernel of the derivation $\delta_{2}$ on the noncommutative torus (since $\delta_{2}\left(U_{1}\right)$ is vanishing). Hence one can add $\delta_{2}$ to $\tau \delta_{1}$ making $\tilde{\nabla}$ a connection on $\tilde{M}$ covering $\delta_{\tau}$.

Note that by a simple adjustment one can actually define a right exact functor from $\mathcal{B}_{q}^{\omega_{1}}$ to $\operatorname{Vect}\left(\mathbb{T}_{\theta}^{\omega}\right)$. We also claim that, in fact, 
Proposition 9. The map $\psi$ endows $\mathcal{A}_{\theta}$ with a flat module structure over $\mathcal{O}\left(\mathbb{C}^{*}\right)$.

Proof. The algebra $\mathcal{O}\left(\mathbb{C}^{*}\right)$ is a commutative integral domain, since holomorphic functions cannot have disjoint support. Further, from Corollary 3.2 of [19] one concludes that the global Ext dimension of $\mathcal{O}\left(\mathbb{C}^{*}\right)$ is 1 . Hence it is a Prüfer domain, i.e., a domain in which all finitely generated non-zero ideals are invertible. Indeed, Theorem 6.1 of [10] says that a (fractional) ideal in a domain is invertible if and only if it is projective and, since $\mathcal{O}\left(\mathbb{C}^{*}\right)$ has Ext dimension 1, given any finitely generated ideal $I$, applying $\operatorname{Hom}(-, M)$ to the exact sequence $0 \rightarrow I \rightarrow R \rightarrow R / I \rightarrow 0$ for an arbitrary $M$, one finds that $\operatorname{Ext}^{1}(I, M)=0$, i.e., $I$ is projective. It is known that a module over a Prüfer domain is flat if and only if it is torsion-free (see, e.g., Theorem 1.4 in [10]). So we only need to check torsion-freeness. We identify $\mathcal{A}_{\theta}$ as a module over $\mathcal{O}\left(\mathbb{C}^{*}\right)$ with $\delta\left(\mathbb{Z}, C^{\infty}\left(\mathbb{S}^{1}\right)\right)$ and represent each element as a sequence $\left\{g_{n}\right\}_{n \in \mathbb{Z}}, g_{n} \in C^{\infty}\left(\mathbb{\$}^{1}\right)$, refer to the discussion in Section 1.1. The image of the map $\psi$ clearly lies in $C^{\infty}\left(\mathbb{S}^{1}\right)$, which is identified with the functions supported at the identity element of $\mathbb{Z}$. In other words, for all $f \in \mathcal{O}\left(\mathbb{C}^{*}\right), \psi(f)$ is of the form $\left\{f_{n}\right\}$, where $f_{n}=0$ unless $n=0$. Now consider any $g=\left\{g_{n}\right\} \in \mathcal{A}_{\theta}$ and suppose that some non-zero $f \in \operatorname{Ann}\left(\left\{g_{n}\right\}\right)$, i.e., $g * \psi(f)=\left\{g_{n} \alpha_{n}\left(f_{0}\right)\right\}=0$. This implies that $g_{n}(z) f_{0}\left(q^{n} z\right)=0$ for all $n,|z|=1$. Being the restriction of a holomorphic function on $\mathbb{C}^{*}, f_{0}\left(q^{n} z\right)$ has a discrete zero set on the unit circle. A smooth function on $\mathbb{S}^{1}$ cannot have a discrete set of points as support and hence each $g_{n}(z)$ must be identically zero. Thus, whenever an element in $\mathcal{A}_{\theta}$ has a non-zero element in its annihilator ideal, the element is itself zero. Hence $\mathcal{A}_{\theta}$ is torsion-free from which the result follows.

Corollary 10. The base change functor $\psi_{*}$ induced by the homomorphism $\psi$ is exact and faithful.

Proof. From the previous proposition we conclude that the functor sends an exact sequence of $\mathcal{O}\left(\mathbb{C}^{*}\right)$-modules to an exact sequence of $\mathcal{A}_{\theta}$-modules and clearly the induced morphisms respect the connections. For the faithfulness, identify each object $M \in \mathscr{B}_{q}^{\tau}$ with $V \otimes \mathcal{O}\left(\mathbb{C}^{*}\right)$ with $V$ a vector space; similarly write $M^{\prime}=V^{\prime} \otimes \mathcal{O}\left(\mathbb{C}^{*}\right)$. A morphism in $\mathcal{B}_{q}^{\tau}$ from $M$ to $M^{\prime}$ is then given by an element in $\operatorname{Hom}_{\mathbb{C}}\left(V, V^{\prime}\right) \otimes_{\mathbb{C}}$ $\mathcal{O}\left(\mathbb{C}^{*}\right)$, whereas a morphism in $\operatorname{Vect}\left(\mathbb{\mathbb { T }}_{\theta}^{\tau}\right)$ between $\tilde{M}$ and $\tilde{M}^{\prime}$ is given by an element in $\operatorname{Hom}_{\mathbb{C}}\left(V, V^{\prime}\right) \otimes_{\mathbb{C}} \mathcal{A}_{\theta}$. The functor $\psi_{*}$ acts on these element by $1 \otimes \psi$ and since $\psi$ is injective, it follows that $\psi_{*}$ is injective on morphisms.

Remark 11. However, the functor is not full. It is certainly not essentially surjective as the underlying $\mathscr{A}_{\theta}$-modules of the objects in the image are all free, whilst $\operatorname{Vect}\left(\mathbb{T}_{\theta}^{\tau}\right)$ has modules which are not free. It is also not injective on objects. 
Remark 12. Before Proposition 6 we introduced the category $\mathscr{B}^{\tau} \simeq \operatorname{Rep}(\mathbb{Z})$ as the category of bundles on $\mathbb{C}^{*}$ with regular singular connections compatible with $\tau z \frac{d}{d z}$. The functor $\psi_{*}$ can actually be regarded as a functor between $\mathscr{B}^{\tau}$ and $\operatorname{Vect}\left(\mathbb{\mathbb { T }}_{\theta}^{\tau}\right)$ and we may precompose it with the forgetful functor $\mathscr{B}_{q}^{\tau} \rightarrow \mathscr{B}^{\tau}$ to get our desired $\psi_{*}: \mathscr{B}_{q}^{\tau} \rightarrow \operatorname{Vect}\left(\mathbb{T}_{\theta}^{\tau}\right)$.

The main theorem of [20] says that the category generated by successive extensions of all standard holomorphic bundles over $\mathbb{T}_{\theta}^{\tau}$ is already all of $\operatorname{Vect}\left(\mathbb{T}_{\theta}^{\tau}\right)$ (refer to Section 1.2 for the definition of standard bundles). Let us denote the full subcategory of $\operatorname{Vect}\left(\mathbb{T}_{\theta}^{\tau}\right)$ generated by successive extensions of standard modules of the form $E_{1}^{z^{\prime}}$, $z^{\prime} \in \mathbb{C}$ by $\operatorname{FrVect}\left(\mathbb{T}_{\theta}^{\tau}\right)$. Since the extension of two free modules is again free, it is clear that the underlying $\mathcal{A}_{\theta}$-module of all objects of $\operatorname{FrVect}\left(\mathbb{T}_{\theta}^{\tau}\right)$ is free.

Lemma 13. With respect to a suitable basis each object of $\operatorname{FrVect}\left(\mathbb{T}_{\theta}^{\tau}\right)$ is of the form $\left(\mathcal{A}_{\theta}^{n}, \delta_{\tau}+A\right)$, where $A$ is an $n \times n$ upper triangular matrix in $M_{n}\left(\mathcal{A}_{\theta}\right)$ with diagonal entries in $\mathbb{C}$.

Proof. It is known that given any finitely generated projective module $M$ over $\mathcal{A}_{\theta}$ and a fixed connection $\nabla$ compatible with $\delta_{\tau}$, all other compatible connections are of the form $\nabla+\phi, \phi \in \operatorname{End}_{\mathcal{A}_{\theta}}(M)$. This follows easily from the Leibniz rule (2). Since $M$ is of the form $\mathcal{A}_{\theta}^{n}, \phi$ is determined by a matrix $A \in M_{n}\left(\mathcal{A}_{\theta}\right)$. Let

$$
0 \rightarrow\left(\mathcal{A}_{\theta}, \nabla_{z^{\prime}}\right) \stackrel{\iota}{\rightarrow}\left(\mathcal{A}_{\theta}^{2}, \delta_{\tau}+A\right) \stackrel{\pi}{\rightarrow}\left(\mathcal{A}_{\theta}, \nabla_{z^{\prime \prime}}\right) \rightarrow 0
$$

be a holomorphic extension in $\operatorname{Vect}\left(\mathbb{T}_{\theta}^{\tau}\right)$. Write $A=\left(\begin{array}{ll}a & b \\ c & d\end{array}\right)$ with entries $a, b, c, d \in$ $\mathcal{A}_{\theta}$ and $\iota(a)=(a, 0), \pi\left(a_{1}, a_{2}\right)=a_{2}$. One checks easily that the holomorphicity of $\iota$ and $\pi$ (the fact that they commute with the connections) forces $c=0, a=z^{\prime}$ and $d=z^{\prime \prime}$. Now by induction it follows that the connections obtained by successive extensions are of the desired form.

Conversely, by induction suppose that every connection of the desired form on $\mathcal{A}_{\theta}^{n-1}$ can be obtained as an iterated extension of modules of the form $E_{1}^{z^{\prime}}$. Let $A$ be an upper triangular matrix in $M_{n}\left(\mathcal{A}_{\theta}\right)$ whose diagonal entries are in $\mathbb{C}$, i.e., $A$ is of the form

$$
\left(\begin{array}{cccc}
z^{\prime} & b_{2} & \cdots & b_{n} \\
0 & & & \\
\vdots & & A^{\prime} & \\
0 & & &
\end{array}\right),
$$

where $A^{\prime} \in M_{n-1}\left(\mathcal{A}_{\theta}\right)$ is also of the prescribed type and $b_{2}, \ldots, b_{n} \in \mathcal{A}_{\theta}$. A routine calculation then shows that

$$
0 \rightarrow\left(\mathcal{A}_{\theta}, \nabla_{z^{\prime}}\right) \stackrel{\iota}{\rightarrow}\left(\mathcal{A}_{\theta}^{n}, \delta_{\tau}+A\right) \stackrel{\pi}{\rightarrow}\left(\mathcal{A}_{\theta}^{n-1}, \delta_{\tau}+A^{\prime}\right) \rightarrow 0,
$$


with $\iota(a)=(a, 0, \ldots, 0)$ and $\pi\left(a_{1}, a_{2}, \ldots, a_{n}\right)=\left(a_{2}, \ldots, a_{n}\right)$ is a holomorphic extension in $\operatorname{Vect}\left(\mathbb{T}_{\theta}^{\tau}\right)$. Hence $\left(\mathcal{A}_{\theta}^{n}, \delta_{\tau}+A\right)$ belongs to $\operatorname{FrVect}\left(\mathbb{T}_{\theta}^{\tau}\right)$.

Remark 14. Given any matrix $A \in M_{n}(\mathbb{C})$, with respect to a suitable basis one can reduce it to its Jordan canonical form (it is also upper triangular with diagonal entries in $\mathbb{C})$. Therefore, $\operatorname{FrVect}\left(\mathbb{T}_{\theta}^{\tau}\right)$ contains all objects of the form $\left(\mathcal{A}_{\theta}^{n}, \delta+A\right)$, where $A \in M_{n}(\mathbb{C})$ with respect to a basis.

As we will see later (Proposition 18), each object $(M, \sigma, \nabla)$ in $\mathscr{B}_{q}^{\tau}$ is isomorphic to an object, whose matrix of the connection is a constant matrix. This can be accomplished via a change of basis of $M$. Combining this with the above remark, we conclude that the image of $\psi_{*}$ is a subcategory $\operatorname{FrVect}\left(\mathbb{\mathbb { T }}_{\theta}^{\tau}\right)$.

2.2. The effect on K-theory. We infer from eqn. (4) that the K-theory (by that we mean the Grothendieck group, i.e., the free abelian group generated by the isomorphism classes of objects modulo the relations coming from all exact sequences) of $\operatorname{Vect}\left(\mathbb{T}_{\theta}^{\tau}\right)$ is isomorphic to that of $D^{b}\left(X_{\tau}\right)$ via the Polishchuk-Schwarz equivalence $\delta_{\tau}$. One knows that $K_{0}\left(D^{b}\left(X_{\tau}\right)\right) \cong K_{0}\left(\operatorname{Coh}\left(X_{\tau}\right)\right)=K_{0}\left(X_{\tau}\right)=\operatorname{Pic}\left(X_{\tau}\right) \oplus \mathbb{Z}$. The composition of the functors $\psi_{*}$ followed by $\delta_{\tau}$ induces a homomorphism between $K_{0}\left(\mathscr{B}_{q}^{\tau}\right)$ and $K_{0}\left(\operatorname{Vect}\left(\mathbb{T}_{\theta}^{\tau}\right)\right)=\operatorname{Pic}\left(X_{\tau}\right) \oplus \mathbb{Z}$. One observes that applying $\psi_{*}$ one obtains only elements in $\operatorname{Vect}\left(\mathbb{T}_{\theta}^{\tau}\right)$ whose underlying $\mathcal{A}_{\theta}$-modules are free. It is known that for $E \in \operatorname{Vect}\left(\mathbb{T}_{\theta}^{\tau}\right), \operatorname{rk} \delta_{\tau}(E)=-\operatorname{deg}(E)$ and $\operatorname{deg} \delta_{\tau}(E)=\operatorname{rk}(E)$. The degree of the modules, which are free, is known to be zero. Hence the composition of the two functors sends every element in $\mathscr{B}_{q}^{\tau}$, whose image under $\psi_{*}$ is a standard bundle, to a torsion sheaf on $X_{\tau}$. One can check that $\mathcal{O}\left(\mathbb{C}^{*}\right)$ equipped with the connection $\delta+z^{\prime}$, where $z^{\prime} \in \mathbb{C}$, gets mapped to the standard holomorphic bundle $E_{1}^{z^{\prime}}$ as explained after Remark 12. From part (c) of Proposition 3.7 of [23] we know that $\boldsymbol{S}_{\tau}\left(E_{1}^{z^{\prime}}\right)$ is $\mathcal{O}_{-z^{\prime}}$ (up to a shift in the derived category), which is the structure sheaf of the point $-z^{\prime}$ mod $(\mathbb{Z}+\tau \mathbb{Z})$ in $X_{\tau}$. All modules of the form $\left(\mathcal{O}\left(\mathbb{C}^{*}\right), \sigma, \delta+z^{\prime}\right)$ with $z^{\prime} \in \mathbb{C}$ are endomorphism simple, i.e., $\operatorname{End}\left(\mathcal{O}\left(\mathbb{C}^{*}\right), \sigma, \delta+z^{\prime}\right)=\mathbb{C}$. Indeed, ignoring the equivariance condition and the connection, $\operatorname{End}\left(\mathcal{O}\left(\mathbb{C}^{*}\right)\right)=\mathcal{O}\left(\mathbb{C}^{*}\right)$ and the equivariance condition says that $\sigma(m f)=\sigma(m) f$. However, by definition $\sigma(m f)=\sigma(m) \alpha(f)$ whence $\alpha(f)=f$ implying $f \in \mathbb{C}$. This module is mapped to $\left(\mathcal{A}_{\theta}, \delta_{\tau}+2 \pi i z^{\prime}\right)=E_{1}^{z^{\prime}}$, which in turn is mapped to the endomorphism simple object $\mathcal{O}_{-z^{\prime}}$ in $\ell^{\theta, \tau}$. It is known that, in fact, the Grothendieck group of any nonsingular curve $C$ is isomorphic to $\operatorname{Pic}(C) \oplus \mathbb{Z}$. In this identification the contribution to $\mathbb{Z}$ comes from the rank of the coherent sheaf, whereas $\operatorname{Pic}(C)$ can be regarded as the contribution from the torsion part (actually from the determinant bundle of the sheaf, which may be identified with a torsion sheaf via a Fourier-Mukai transform). Since we shall see later on (Corollary 21) that the classes of $\left(\mathcal{O}\left(\mathbb{C}^{*}\right), \sigma, \delta+z^{\prime}\right)$ generate the K-theory of $\mathscr{B}_{q}^{\tau}$, the image of the induced map on K-theory lies inside $\operatorname{Pic}\left(X_{\tau}\right)$. 
Proposition 15. The map induced by $\wp_{\tau} \circ \psi_{*}$ between the K-theories of $\mathcal{B}_{q}^{\tau}$ and $\operatorname{Vect}\left(\mathbb{\nabla}_{\theta}^{\tau}\right)$ gives a surjection from $K_{0}\left(\mathscr{B}_{q}^{\tau}\right)$ to $\operatorname{Pic}\left(X_{\tau}\right)$.

Proof. The divisor class group of $X_{\tau}$ is the free abelian group generated by the points of $X_{\tau}$ modulo the principal divisors, which is also isomorphic to $\operatorname{Pic}\left(X_{\tau}\right)$. The class of each point $z^{\prime} \in X_{\tau}$ of the divisor class group can be identified with the class of the torsion sheaf $\mathcal{O}_{z^{\prime}}$ corresponding to the line bundle $\mathcal{O}\left(z^{\prime}\right) \in \operatorname{Pic}^{1}\left(X_{\tau}\right)$ and they generate $\operatorname{Pic}\left(X_{\tau}\right)$ as a group. By the above argument $\mathcal{O}_{z^{\prime}}$ is obtained by applying the functor $\delta_{\tau} \circ \psi_{*}$ to the element $\left(\mathcal{O}\left(\mathbb{C}^{*}\right), \sigma, \delta-z^{\prime}\right)$ of $\mathcal{B}_{q}^{\tau}$. Thus one obtains a surjection onto the generating set of $\operatorname{Pic}\left(X_{\tau}\right)$ from which the assertion follows.

Remark 16. From Proposition 2.1 of [23] we know that the images of $\left(\mathcal{O}\left(\mathbb{C}^{*}\right), \sigma, \delta+z_{1}^{\prime}\right)$ and $\left(\mathcal{O}\left(\mathbb{C}^{*}\right), \sigma, \delta+z_{2}^{\prime}\right)$ under $\psi_{*}$ are isomorphic if and only if $z_{1}^{\prime} \equiv z_{2}^{\prime} \bmod (\mathbb{Z}+\tau \mathbb{Z})$. More generally, abbreviating the module $\left(\mathcal{O}\left(\mathbb{C}^{*}\right), \sigma, \delta+z^{\prime}\right)$ by $M_{z^{\prime}}$, one can also rephrase the linear equivalence relation of the divisor class group to conclude that an element of the form $\sum n_{i}\left[M_{-z_{i}^{\prime}}\right]$ maps to zero at the level of Ktheory whenever $\sum n_{i}=0$ and $\sum n_{i} z_{i}^{\prime} \in(\mathbb{Z}+\tau \mathbb{Z})$. However, some of them actually represent the trivial class in the K-theory of $\mathscr{B}_{q}^{\tau}$, as we will see in the next section (see Corollary 21).

Although the image of $\mathcal{B}_{q}^{\tau}$ gives only the free modules in $\operatorname{Vect}\left(\mathbb{T}_{\theta}^{\tau}\right)$, it has the interesting property of being a Tannakian category, as we will explore in the next section. Let us end this section by summarising the relations between $\mathcal{B}_{q}^{\tau}$ and the categories $\mathcal{B}^{\tau}, \mathcal{B}_{q}, \operatorname{Vect}\left(\mathbb{T}_{\theta}^{\tau}\right)$ :

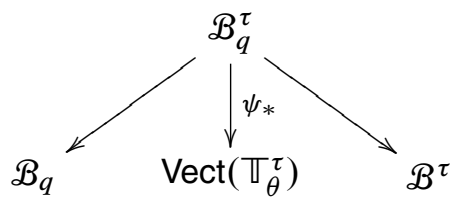

where the two diagonal arrows are the forgetful functors discussed before. All of these functors are faithful and exact.

\section{The Tannakian formalism and the equivariant Riemann-Hilbert correspondence}

We will now analyse further the structure of $\mathcal{B}_{q}^{\tau}$ and define a tensor product on it. Our main result is that this - together with a fibre functor - makes $\mathcal{B}_{q}^{\tau}$ a Tannakian category. Via an equivariant version of the Riemann-Hilbert correspondence on $\mathbb{C}^{*}$, we determine the corresponding affine group scheme. 
3.1. Preliminaries on Tannakian categories. We briefly recall the notion of a Tannakian category. For more details, we refer the reader to the original works [29], [7], [8] (see also Appendix B of [25]).

Let $\mathcal{C}$ be an $k$-linear abelian category, for a field $k$. Then $\mathcal{C}$ is a neutral Tannakian category over $k$ if the following holds:

(1) The category $C$ is a tensor category. In other words, there is a tensor product: for every pair of objects $X, Y$ there is an object $X \otimes Y$. The tensor product is commutative $X \otimes Y \simeq Y \otimes X$ and associative $X \otimes(Y \otimes Z) \simeq(X \otimes Y) \otimes Z$ and there is a unit object 1 (such that $X \otimes 1 \simeq 1 \otimes X \simeq X$ ). The above isomorphisms are supposed to be functorial.

(2) $\mathcal{C}$ is a rigid tensor category: there exists a duality $\vee: \mathcal{\ell} \rightarrow \ell^{\mathrm{op}}$ such that:

- For any object $X$ in $\mathcal{C}$, the functor ${ }_{-} \otimes X^{\vee}$ is left adjoint to ${ }_{-} \otimes X$, and the functor $X^{\vee} \otimes_{-}$is right adjoint to $X \otimes_{-}$.

- There is an evaluation morphism $\epsilon: X \otimes X^{\vee} \rightarrow 1$ and a unit morphism $\eta: 1 \rightarrow X^{\vee} \otimes X$ such that $(\epsilon \otimes 1) \circ(1 \otimes \eta)=1_{X}$ and $(1 \otimes \epsilon) \circ(\eta \otimes 1)=1_{X^{\vee}}$.

(3) An isomorphism between End(1) and $k$ is given.

(4) There is a fibre functor $\omega: \mathcal{C} \rightarrow$ Vect $_{k}$ to the category of $k$-vector spaces: this is a $k$-linear, faithful, exact functor that commutes with tensor products.

In general, the fibre functor could be $L$-valued, where $L$ is a field extension of $k$. Henceforth, unless otherwise stated, we shall consider only neutral Tannakian categories, i.e., those with a $k$-valued fibre functor. An important result is that every Tannakian category is equivalent to the category of finite dimensional linear representations of an affine group scheme $G$ over $k$. Abstractly, it is given by the automorphism group scheme of the fibre functor. However, in most examples in the literature, a concrete equivalence with $\operatorname{Rep}(G)$ for some $G$ is established, and we will do so as well.

3.2. The Tannakian category structure on $\boldsymbol{B}_{\boldsymbol{q}}^{\boldsymbol{\tau}}$. Let us generalize a little and let $(R, \delta)$ be a differential (commutative) ring that carries an action $\alpha$ of a group $G$. Let $\operatorname{Mod}^{G, \delta}(R)$ denote the category consisting of free $G$-equivariant differential $R$ modules. Recall that a differential $R$-module is an $R$-module equipped with a map $\nabla: M \rightarrow M-$ a connection - that satisfies the Leibniz rule

$$
\nabla(m \cdot r)=\nabla(m) \cdot r+m \cdot \delta(r) .
$$

Moreover, $G$-equivariance means that there is an action $\sigma$ of $G$ such that

$$
\begin{aligned}
\sigma_{g}(m \cdot r) & =\sigma_{g}(m) \cdot \alpha_{g}(r), \\
\nabla\left(\sigma_{g}(m)\right) & =\sigma_{g}(\nabla(m)) .
\end{aligned}
$$


We will group the objects in the category $\operatorname{Mod}^{G, \delta}(R)$ into a triple $(M, \sigma, \nabla)$ and denote the morphisms that respect all the structures by $\operatorname{Hom}_{R}^{G, \delta}(M, N)$.

Proposition 17. The category $\operatorname{Mod}^{G, \delta}(R)$ is a rigid tensor category with the tensor product given by

$$
(M, \sigma, \nabla) \otimes\left(N, \sigma^{\prime}, \nabla^{\prime}\right)=\left(M \otimes \otimes_{\mathcal{O}\left(\mathbb{C}^{*}\right)} N, \sigma \otimes \sigma^{\prime}, \nabla \otimes 1+1 \otimes \nabla^{\prime}\right)
$$

for any two objects $(M, \sigma, \nabla)$ and $\left(N, \sigma^{\prime}, \nabla^{\prime}\right)$ in $\operatorname{Mod}^{G, \delta}(R)$.

Proof. We start by checking that the tensor product is commutative. First of all, since $R$ is a commutative ring, the 'tensor flip' that maps $M \otimes_{\mathbb{C}} N \rightarrow N \otimes_{\mathbb{C}} M$ factorizes to a bijective map of $R$-modules from $M \otimes_{R} N$ to $N \otimes_{R} M$. One also checks that it intertwines the actions $\sigma \otimes \sigma^{\prime}$ and $\sigma^{\prime} \otimes \sigma$ and the two connections.

The duality is given as follows, for an object $(M=V \otimes R, \sigma, \nabla), V$ a vector space, we define its dual object $\left(M^{\vee}, \sigma^{\vee}, \nabla^{\vee}\right)$ as follows. Define an $R$-module by

$$
M^{\vee}:=\operatorname{Hom}_{R}(M, R),
$$

with $r \in R$ acting on $f \in M^{\vee}$ by $(f \cdot r)(m)=f(m) \cdot r=f(m \cdot r)$. It can be equipped with a dual action $\sigma^{\vee}$ of $G$ by setting for $f \in M^{\vee}$,

$$
\sigma^{\vee}(f)=\alpha \circ f \circ \sigma^{-1} \text {. }
$$

One can check that $\sigma^{\vee}(f)$ is again $R$-linear:

$\sigma^{\vee}(f)(m \cdot r)=\alpha \circ f\left(\sigma^{-1}(m) \cdot \alpha^{-1}(r)\right)=\alpha \circ f \circ \sigma^{-1}(m) \cdot r=\left(\sigma^{\vee}(f) \cdot r\right)(m)$.

Moreover, the action of $R$ on $M^{\vee}$ is equivariant with respect to $\sigma^{\vee}$ :

$\sigma^{\vee}(f \cdot r)(m)=\alpha \circ(f \cdot r)\left(\sigma^{-1}(m)\right)=\alpha\left(f\left(\sigma^{-1}(m)\right) \cdot r\right)=\alpha \circ f \circ \sigma^{-1}(m) \cdot \alpha(r)$.

A dual connection $\nabla^{\vee}$ is defined by

$$
\nabla^{\vee}(f)=\delta \circ f-f \circ \nabla,
$$

which indeed satisfies the Leibniz rule

$$
\begin{aligned}
\nabla^{\vee}(f \cdot r)(m) & =\delta(f(m)) \cdot r+f(m) \cdot \delta(r)-f(\nabla(m)) \cdot r \\
& =\left(\nabla^{\vee}(f) \cdot r\right)(m)+(f \cdot \delta(r))(m),
\end{aligned}
$$

and is $\sigma^{\vee}$-invariant:

$\sigma^{\vee}\left(\nabla^{\vee}(f)\right)=\alpha \circ(\delta \circ f) \circ \sigma^{-1}-\alpha \circ(f \circ \nabla) \circ \sigma^{-1}=\delta \circ\left(\alpha \circ f \circ \sigma^{-1}\right)-\left(\alpha \circ f \circ \sigma^{-1}\right) \circ \nabla$,

since $\alpha$ and $\sigma$ commute with $\delta$ and $\nabla$, respectively. 
Note that since $M=V \otimes R$, we can identify,

$$
M^{\vee} \simeq \operatorname{Hom}_{R}(V \otimes R, R) \simeq \operatorname{Hom}_{\mathbb{C}}(V, \mathbb{C}) \otimes R \simeq V^{*} \otimes R,
$$

from which it follows that $M^{\vee \vee} \simeq M$. Indeed, one checks that the induced map respects the extra $(G, \delta)$-structure:

$$
\begin{aligned}
\sigma^{\vee \vee}(m)(f) & =\alpha \circ m \circ\left(\sigma^{\vee}\right)^{-1}(f)=\alpha \circ m \circ\left(\alpha^{-1} \circ f \circ \sigma\right)=f(\sigma(m)), \\
\nabla^{\vee \vee}(m)(f) & =(\delta \circ m)(f)-m \circ \nabla^{\vee}(f) \\
& =\delta(f(m))-m \circ(\delta \circ f)+f(\nabla(m))=f(\nabla(m))
\end{aligned}
$$

for all $m \in M, f \in M^{\vee}$. In addition, it allows one to prove that the association

$$
\begin{aligned}
\phi \in \operatorname{Hom}_{R}^{G, \delta}\left(N_{1}, M^{\vee} \otimes_{R} N_{2}\right) & \mapsto \tilde{\phi} \in \operatorname{Hom}_{R}^{G, \delta}\left(M \otimes_{R} N_{1}, N_{2}\right), \\
\tilde{\phi}\left(m \otimes n_{1}\right) & :=\phi\left(n_{1}\right)(m) \in N_{2} .
\end{aligned}
$$

induces an isomorphism. Again, it is enough to show that this map is both $G$ equivariant and $\delta$-invariant, which is left as an exercise.

In a similar way, one proves that

$$
\operatorname{Hom}_{R}^{G, \delta}\left(N_{1} \otimes_{R} M^{\vee}, N_{2}\right) \simeq \operatorname{Hom}_{R}^{G, \delta}\left(N_{1}, N_{2} \otimes_{R} M\right) .
$$

Finally, there is an evaluation morphism and a unit morphism given in terms of a basis $\left\{e_{i}\right\}$ of $V$ and its dual $\left\{\hat{e}_{i}\right\}$ of $V^{*}$ by

$$
\epsilon(m \otimes f)=f(m), \quad \eta\left(1_{R}\right)=\hat{e}_{i} \otimes e_{i},
$$

which satisfy the required properties.

Let us now return to the category $\mathscr{B}_{q}^{\tau}$ of Definition 4 . It is not difficult to see that the above tensor product respects the regular singularity condition in the definition of $\mathcal{B}_{q}^{\tau}$. Hence this becomes a rigid tensor category as well. We would like to show that it is in fact a Tannakian category by constructing a fibre functor to Vect $\mathbb{C}$. The following observations turn out to be essential in what follows.

Via a series of changes of basis, it is possible to bring the matrix $A$ in the form of a constant matrix with all eigenvalues in the same transversal of $\tau \mathbb{Z}$. In other words, its eigenvalues never differ by an integer multiple of $\tau$. We follow the argument of Section 17 in [32]. Let $A(z)=A_{0}+A_{1} z+\cdots$ be a matrix with holomorphic entries. We first bring the constant term $A_{0}$ in Jordan canonical form via a constant change of basis matrix. Subsequently, we can bring all the eigenvalues of $A_{0}$ in the same transversal of $\tau \mathbb{Z}$ by the so-called shearing transformations. Let us consider the case of a $2 \times 2$ matrix $A(z)$ and write

$$
A(z)=\left(\begin{array}{cc}
\lambda_{1} & 0 \\
0 & \lambda_{2}
\end{array}\right)+\left(\begin{array}{ll}
a(z) & b(z) \\
c(z) & d(z)
\end{array}\right)
$$


with $a=a_{1} z+a_{2} z^{2}+\cdots$ and similarly $b, c$ and $d$. Let us suppose that $\lambda_{1}-\lambda_{2}=k \tau$ for some positive integer $k$. The change of basis is given by the matrix $D=\operatorname{diag}\{1, z\}$ and transforms $A$ to

$$
A^{\prime}=D^{-1} A D+D^{-1} \delta D=\left(\begin{array}{cc}
\lambda_{1} & 0 \\
c_{1} & \lambda_{2}+\tau
\end{array}\right)+\left(\begin{array}{cc}
a(z) & z b(z) \\
c_{2} z+c_{3} z^{2}+\cdots & d(z)
\end{array}\right),
$$

and one readily observes that the constant term $A_{0}^{\prime}$ of this matrix has eigenvalues that differ by $(k-1) \tau$. Proceeding in this way, one can transform $A$ to a matrix that has constant term with eigenvalues in the same transversal. The generalization to arbitrary dimensions is straightforward and can be found in Section 17.1 of [32].

Proposition 18. For each object in $\mathscr{B}_{q}^{\tau}$, there exists an isomorphic object $\left(M=V \otimes \mathcal{O}\left(\mathbb{C}^{*}\right), \sigma, \nabla\right)$ in $\mathscr{B}_{q}^{\tau}$ with $V$ a vector space and

(1) $\nabla=\delta+A$ with $A$ a constant matrix with all eigenvalues in the same transversal of $\tau \mathbb{Z}$,

(2) $\sigma$ is given by $\sigma(v \otimes f)=B v \otimes \alpha(f)$ for an invertible constant matrix $B$.

Proof. Since $M$ is a free $\mathcal{O}\left(\mathbb{C}^{*}\right)$-module, there is a vector space $V$ such that $M \simeq$ $V \otimes \mathcal{O}\left(\mathbb{C}^{*}\right)$. We show (1) by adopting an argument from Section 5 of [32]. By the above observations, we can write the matrix of the connection as $A=A_{0}+A_{1} z+\cdots$, with $A_{0}$ having eigenvalues that never differ by an element of $\tau \mathbb{Z}$. We construct a matrix $P=\mathrm{I}+P_{1} z+\cdots\left(P_{k}\right.$ in $\left.M_{n}(\mathbb{C})\right)$ which solves $P A_{0}=A P-\delta P$. Comparing the powers of $z$, we find that

$$
A_{0} P_{k}-P_{k}\left(A_{0}+\tau k \mathrm{I}\right)=-\left(A_{k}+A_{k-1} P_{1}+\cdots+A_{1} P_{k-1}\right),
$$

which can be solved recursively by our assumption on the eigenvalues of $A_{0}$. This gives a formal power series expansion and we would like to show that the entries of $P$ are in fact holomorphic functions on $\mathbb{C}^{*}$.

Now by Theorem 5.4 of [32] one knows that the radius of convergence of the entries of $P$ is the same as that of the entries of $A$, which is infinity. Hence, $P \in M_{n}\left(\mathcal{O}\left(\mathbb{C}^{*}\right)\right)$.

Next, the action of $\sigma$ can be written as $\sigma(v \otimes f)=B v \otimes \alpha(f)$ for some invertible matrix $B \in M_{n}\left(\mathcal{O}\left(\mathbb{C}^{*}\right)\right)$ with $n$ the dimension of $V$. Expressed in terms of $A$ and $B$, the equivariance condition $\sigma \circ \nabla=\nabla \circ \sigma$ reads

$$
\delta B+[A, B]=0,
$$

and as observed above, we may assume that $A$ has constant entries and with eigenvalues that are all in the same transversal. We adopt the argument from the proof of Theorem 4.4 in [14] to show that $B$ is in fact constant. Writing $B$ as a Laurent series $B=\sum_{k \in \mathbb{Z}} B_{k} z^{k}$ we obtain the relations

$$
\left(A-\tau k \mathrm{I}_{n}\right) B_{k}=B_{k} A, \quad k \in \mathbb{Z} .
$$


This implies ([32], Theorem 4.1; see also Lemma 4.6 in [14]) that $\left(A-\tau k \mathrm{I}_{n}\right)$ and $A$ have at least one common eigenvalue. But since the eigenvalues of $A$ are all in a transversal of $\tau \mathbb{Z}$ in $\mathbb{C}$, this is impossible unless $k=0$, and we conclude that $B_{k}=0$ for all $k \neq 0$.

Our next task is to show that $\mathscr{B}_{q}^{\tau}$ is in fact a Tannakian category and compute the corresponding affine group scheme. For this, we use an equivariant version of the Riemann-Hilbert correspondence.

Theorem 19. (1) The category $\mathscr{B}_{q}^{\tau}$ is a Tannakian category with the fibre functor given by

$$
\omega: \mathcal{B}_{q}^{\tau} \rightarrow \operatorname{Vect}_{\mathbb{C}}, \quad(M, \sigma, \nabla) \mapsto(\operatorname{ker} \nabla)_{z},
$$

mapping an object in $\mathcal{B}_{q}^{\tau}$ to the germs at a fixed point $z \in \mathbb{C}^{*}$ of local solutions to the differential equation $\delta f+A f=0$, where $\nabla=\delta+A$ with respect to a suitable basis of $M$.

(2) The category $\mathscr{B}_{q}^{\tau}$ is equivalent to the category $\operatorname{Rep}(\mathbb{Z}+\theta \mathbb{Z})$ of finite dimensional representations of $\mathbb{Z}+\theta \mathbb{Z} \simeq \mathbb{Z}^{2}$.

Proof. By the existence and uniqueness of local solutions of linear differential equations, there are $n$ local solutions to the system of differential equations $\delta U=-A U$ once we have fixed the initial conditions, so that $(\operatorname{ker} \nabla)_{z}$ is an $n$-dimensional complex vector space. That the functor $\omega$ is faithful can be seen as follows. Suppose $\phi$ is a morphism between two objects $(M, \sigma, \nabla)$ and $\left(M^{\prime}, \sigma^{\prime}, \nabla^{\prime}\right)$ and suppose that these objects are of the form as in Proposition 18, with the eigenvalues of $A, A^{\prime}$ in the same transversal. We claim that $\phi$ is given by a constant matrix so that $\omega(\phi)$ mapping $(\operatorname{ker} \nabla)_{z}$ to $\left(\operatorname{ker} \nabla^{\prime}\right)_{z}$ coincides with $\phi$. The argument is very similar to that used in the second part of the proof of Proposition 18 since compatibility of $\phi$ with the connections implies that

$$
\left(A^{\prime}-\tau k \mathrm{I}_{n}\right) \phi_{k}=\phi_{k} A, \quad k \in \mathbb{Z},
$$

where we have written $\phi=\sum_{k \in \mathbb{Z}} \phi_{k} z^{k}$. An application of Theorem 4.1 in [32] then implies that $A$ and $A^{\prime}-\tau k \mathrm{I}_{n}$ have a common eigenvalue. This is impossible unless $k=0$ since by assumption $A$ and $A^{\prime}$ have eigenvalues in the same transversal. We conclude that $\phi_{k}=0$ for all $k \neq 0$ so $\phi$ is given by a constant matrix, intertwining $A$ and $A^{\prime}$.

The general case follows by observing that Proposition 18 implies that a morphism between two objects in $\mathcal{B}_{q}^{\tau}$ can always be written as $D_{2} \circ \phi \circ D_{1}^{-1}$ with $\phi$ constant as above and with $D_{i}$ certain (invertible) change of basis matrices.

As regards (2), fix a transversal $\boldsymbol{T}$ to $\tau \mathbb{Z}$ in $\mathbb{C}$. We construct a tensor functor $\mathrm{F}_{\boldsymbol{T}}: \operatorname{Rep}\left(\mathbb{Z}^{2}\right) \rightarrow \mathcal{B}_{q}^{\tau}$ that is full, faithful and essentially surjective. Let $\rho_{1}, \rho_{2}$ be two mutually commuting representations of $\mathbb{Z}$ on a vector space $V$. Then we define 
$A \in \operatorname{End}(V)$ via $\rho_{1}(1)=e^{2 \pi i A / \tau}$ and $B$ as $\rho_{2}(1)$. By Lemma 4.5 in [14], there exists a unique matrix $A$ such that $\rho_{1}(1)=e^{2 \pi i A / \tau}$ with its eigenvalues in the transversal $\boldsymbol{T}$ and a unique matrix $B^{\prime}$ such that $B=e^{2 \pi i B^{\prime}}$. We set $\mathrm{F}_{\boldsymbol{T}}(V)=(M, \sigma, \nabla)$ in $\mathcal{B}_{q}^{\tau}$ by putting $M=V \otimes \mathcal{O}\left(\mathbb{C}^{*}\right), \sigma(v \otimes f)=B v \otimes \alpha(f)$ and finally $\nabla(v \otimes f)=$ $A v \otimes f+v \otimes \delta f$; for a morphism $\phi \in \operatorname{Hom}\left(V, V^{\prime}\right)$ we simply set $\mathrm{F}_{\boldsymbol{T}}(\phi)=\phi \otimes 1$. Once again by Lemma 4.5 in [14], the matrices $A$ and $B^{\prime}$ commute, whence $A$ and $B=e^{2 \pi i B^{\prime}}$ commute. Thus the compatibility condition between $\sigma$ and $\nabla$ given by eqn. (5) is satisfied. Moreover, $\mathbf{F}_{\boldsymbol{T}}(\phi)$ is compatible with $\sigma$ and $\nabla$ and thus a morphism in $\mathscr{B}_{q}^{\tau}$.

We infer from Proposition 18 that the functor $F_{\boldsymbol{T}}$ is essentially surjective, since any object in $\mathcal{B}_{q}^{\tau}$ is isomorphic to an object obtained from an element in $\operatorname{Rep}\left(\mathbb{Z}^{2}\right)$ by the above procedure.

Fullness and faithfulness of this functor can be seen as follows. Let $V, V^{\prime}$ be two vector spaces with the action of $\mathbb{Z}^{2}$ given by $e^{2 \pi i A / \tau}, B$ and $e^{2 \pi i A^{\prime} / \tau}, B^{\prime}$, respectively. We can choose the square matrices $A$ and $A^{\prime}$ such that their eigenvalues lie in the transversal $\boldsymbol{T}$. It then follows by the same reasoning as before that an element $\rho \in$ $\operatorname{Hom}_{\mathcal{O}\left(\mathbb{C}^{*}\right)}^{\theta \forall \mathbb{R}}\left(M, M^{\prime}\right)$ is given by a constant matrix that intertwines $A, B$ and $A^{\prime}, B^{\prime}$, respectively. Hence, it is given by an element in $\operatorname{Hom}\left(V, V^{\prime}\right)$ that commutes with $\rho_{1}$ and $\rho_{2}$ (i.e., a morphism in $\operatorname{Rep}\left(\mathbb{Z}^{2}\right)$ ).

Finally we show that $F_{\boldsymbol{T}}$ is a tensor functor. Suppose that $\left(V, \rho_{1}, \rho_{2}\right),\left(V^{\prime}, \rho_{1}^{\prime}, \rho_{2}^{\prime}\right)$ are two objects in $\operatorname{Rep}\left(\mathbb{Z}^{2}\right)$; we need to show that there are natural isomorphisms $c_{V, V^{\prime}}: F(V) \otimes F\left(V^{\prime}\right) \rightarrow F\left(V \otimes V^{\prime}\right)$. As before, we define the connection matrix $A$ by setting $e^{2 \pi i A / \tau}=\rho_{1}(1)$ and $B=\rho_{2}(1)$; in the same manner we define $A^{\prime}$ and $B^{\prime}$ from $\rho_{1}^{\prime}$ and $\rho_{2}^{\prime}$. We then have

$$
\begin{aligned}
& F\left(V, \rho_{1}, \rho_{2}\right) \otimes F\left(V^{\prime}, \rho_{1}^{\prime}, \rho_{2}^{\prime}\right) \\
& \quad=\left(\left(V \otimes \mathcal{O}\left(\mathbb{C}^{*}\right)\right) \otimes_{\mathcal{O}\left(\mathbb{C}^{*}\right)}\left(V^{\prime} \otimes \mathcal{O}\left(\mathbb{C}^{*}\right)\right), \sigma \otimes \sigma^{\prime}, \delta+A \otimes 1+1 \otimes A^{\prime}\right) .
\end{aligned}
$$

One observes that the eigenvalues of the matrix $A \otimes 1+1 \otimes A^{\prime}$ lie possibly outside the transversal $\boldsymbol{T}$. However, there is a unique matrix $\tilde{A}$ with all its eigenvalues in $\boldsymbol{T}$ such that

$$
e^{2 \pi i \tilde{A} / \tau}:=e^{2 \pi i\left(A \otimes 1+1 \otimes A^{\prime}\right) / \tau}=e^{2 \pi i A / \tau} \otimes e^{2 \pi i A^{\prime} / \tau} \equiv \rho_{1}(1) \otimes \rho_{1}^{\prime}(1) .
$$

The procedure of associating to $A \otimes 1+1 \otimes A^{\prime}$ the matrix $\tilde{A}$ defines the required map $c_{V, V^{\prime}}$ since $\tilde{A}$ is the connection matrix that one would have obtained (via $\mathrm{F}_{\boldsymbol{T}}$ ) from $\rho_{1} \otimes \rho_{1}^{\prime}$. In fact, it follows that if $A \otimes 1+1 \otimes A^{\prime}$ commutes with $B \otimes B^{\prime} \equiv \rho_{2}(1) \otimes \rho_{2}^{\prime}(1)$ then so does $\tilde{A}$. This map is natural in $V$ and $V^{\prime}$ and the usual diagrams expressing associativity and commutativity (cf. for instance [8], Definition 1.8) are satisfied. Moreover, it is bijective since an inverse can be constructed from eqn. (6) by using the identification $\operatorname{End}_{\mathbb{C}}\left(V \otimes V^{\prime}\right)=\operatorname{End}_{\mathbb{C}}(V) \otimes \operatorname{End}_{\mathbb{C}}\left(V^{\prime}\right)$ to obtain $A$ and $A^{\prime}$ back from $\tilde{A}$. 
Note that the choice of the transversal $\boldsymbol{T}$ is irrelevant since two functors $\mathrm{F}_{\boldsymbol{T}}$ and $\mathrm{F}_{\boldsymbol{T}^{\prime}}$ associated to two different transversals $\boldsymbol{T}$ and $\boldsymbol{T}^{\prime}$ to $\tau \mathbb{Z}$ are related via a natural transformation that is given explicitly by a shearing transformation as discussed before Proposition 18.

We observe that it is also possible to prove the above equivalence directly by means of the fibre functor $\omega$. For this we consider the full subcategory of $\mathcal{B}_{q}^{\tau}$ such that the connection matrices have all eigenvalues in the same transversal $\boldsymbol{T}$. It follows from Proposition 18 that this category is equivalent to $\mathcal{B}_{q}^{\tau}$. By constructing the maps $c_{M, M^{\prime}}$ very similar to those appearing in the above proof, one can show that this is an equivalence of rigid tensor categories. Moreover, the restriction of the fibre functor gives it the structure of a Tannakian category. The fibre functor induces an equivalence with $\operatorname{Rep}\left(\mathbb{Z}^{2}\right)$ by defining the action of $\mathbb{Z}^{2}$ on $(\operatorname{ker} \nabla)_{z}$ to be given by the matrices $e^{2 \pi i A / \tau}$ and $B$. Clearly, the functor $F_{\boldsymbol{T}}$ from the proof of Theorem 19 is the inverse to this fibre functor.

Remark 20. For any group $H$ the category of its finite dimensional representations over $\mathbb{C}$ forms a neutral Tannakian category, which should be equivalent to the category of representations of some affine group scheme, say $\hat{H}$. The group scheme $\widehat{H}$ is called the algebraic hull of $H$. Strictly speaking, the affine group scheme underlying $\mathcal{B}_{q}^{\tau}$ is the algebraic hull of $\mathbb{Z}^{2}$. We refer the readers to Proposition 10.1 of [24] for an explicit computation of the algebraic hull of $\mathbb{Z}$.

As a consequence we are able to conclude that the K-theory of $\mathcal{B}_{q}^{\tau}$ is the same as that of $\operatorname{Rep}\left(\mathbb{Z}^{2}\right)$. An object of $\operatorname{Rep}\left(\mathbb{Z}^{2}\right)$ is a vector space $V$ equipped with two commuting linear invertible endomorphisms. Using the fact that the two endomorphisms commute, i.e., respect each others eigenspaces, one can always find a common eigenvector $w$. This gives an exact sequence $0 \rightarrow\langle w\rangle \rightarrow V \rightarrow V /\langle w\rangle \rightarrow 0$ in $\operatorname{Rep}\left(\mathbb{Z}^{2}\right)$. Therefore, the K-theory of $\operatorname{Rep}\left(\mathbb{Z}^{2}\right)$ is the free abelian group generated by the simple objects, which are one dimensional representations with two actions $a$ and $b$, with $a, b \in \mathbb{C}^{*}$ (the actions are given by multiplication by $a$ and $b$ respectively). The fibre functor sends the isomorphism class of $\left(\mathcal{O}\left(\mathbb{C}^{*}\right), b \alpha, \delta+z^{\prime}\right)$ with $z^{\prime} \in \mathbb{C}$ to the simple object $\left(\mathbb{C}, b, e^{2 \pi i z^{\prime} / \tau}\right)$ in $\operatorname{Rep}\left(\mathbb{Z}^{2}\right)$. Note that $\left(\mathcal{O}\left(\mathbb{C}^{*}\right), b \alpha, \delta+z^{\prime}\right)$ and $\left(\mathcal{O}\left(\mathbb{C}^{*}\right), b \alpha, \delta+\left(z^{\prime}+n \tau\right)\right)$ are isomorphic via the shearing transformation by $z^{n}$. Indeed,

$$
\left(\delta+z^{\prime}\right) z^{n} f=n \tau z^{n} f+z^{n} \delta f+z^{\prime} z^{n} f=z^{n}\left(\delta+\left(z^{\prime}+n \tau\right)\right) f,
$$

and their images also get identified via the exponentiation. Summarising, we obtain

Corollary 21. The K-theory of $\mathfrak{B}_{q}^{\tau}$ is the free abelian group generated by the isomorphism classes of the objects $\left(\mathcal{O}\left(\mathbb{C}^{*}\right), b, \delta+z^{\prime}\right)$ with $b \in \mathbb{C}^{*}$ and $z^{\prime} \in \mathbb{C} / \tau \mathbb{Z}$. Under this identification, one finds that the map on K-theory induced by the functor $\delta_{\tau} \circ \psi_{*}$ sends the class of $\left(\mathcal{O}\left(\mathbb{C}^{*}\right), b, \delta+z^{\prime}\right)$ to the divisor class of the point $-z^{\prime} \in X_{\tau}$ and their linear combinations accordingly. 


\section{4. Étale fundamental group of $\mathbb{T}_{\theta}^{\tau}$}

In the context of noncommutative algebraic geometry typically one treats a small triangulated category with some geometric conditions (like finite total homological dimension) as a noncommutative space (see e.g., [12]). One views a classical smooth and proper algebraic variety in this setting via its bounded derived category of coherent sheaves. Roughly, Nori's notion of an étale fundamental group of a smooth and proper variety relies on realizing a Tannakian category structure on the subcategory of Nori finite bundles of the category of degree 0 semistable bundles [16], [17]. We intend to formulate a similar subcategory in $\operatorname{Vect}\left(\mathbb{T}_{\theta}^{\tau}\right)$ as a proposal for an étale fundamental group of the noncommutative torus. Let us briefly recall the (classical) algebraic setup.

A bundle $\mathcal{F}$ on a smooth and proper algebraic variety $X$ is called finite if its class $[\mathscr{F}] \in K(X)$, where $K(X)$ is the Grothendieck group of the additive monoid of vector bundles on $X$, is integral over $\mathbb{Z}$. The Nori finite bundles are subquotients of finite directs sums of finite bundles. However, in characteristic 0 Nori finite bundles are the same as finite bundles.

Bridgeland's notion of a stability condition on an abstract triangulated category provides an axiomatic characterization of the subcategory of semistable bundles, which are sliced by their phases, a notion closely related to the degree of a bundle (in fact analogous to its slope) [2]. A stability condition on an abstract triangulated category $\mathcal{C}$ is a family of full additive subcategories $\mathcal{P}(\theta)$ parametrized by $\theta \in \mathbb{R}$ and group homomorphism from $\mathcal{Z}: K_{0}(\mathcal{C}) \rightarrow \mathbb{C}$ satisfying a few conditions that can be found in [2]. It turns out that specifying a stability condition is equivalent to specifying a bounded $t$-structure and a group homomorphism from the Grothendieck group of its heart to the complex numbers, sending the effective cone of the Grothendieck group to the upper half plane and satisfying the so-called Harder-Narasimhan property (Proposition 5.3 in [2]).

The objects of $\mathcal{P}(\theta)$ are called semistable objects of phase $\theta$. This definition was motivated by an attempt to understand the "stringy Kähler moduli" space of a Calabi-Yau manifold and so it is expected that a triangulated category equipped with a stability condition should behave like a noncommutative Kähler manifold. As a by-product we get hold of the subcategory of semistable objects $\mathcal{P}_{e}:=\bigcup_{\theta \in \mathbb{R}} \mathcal{P}(\theta)$, which comes with its natural slicing given by $\theta \in \mathbb{R}$. It follows that each subcategory $\mathcal{P}(\theta)$ is abelian (Lemma 5.2 in [2]).

Example 1. Let us look at the bounded derived category of coherent sheaves on a smooth projective curve $X$ denoted by $D^{b}(X)$, which Bridgeland used as an instructive example. We choose the standard $t$-structure on $D^{b}(X)$ and for any $\mathscr{F} \in D^{b}(X)$ denote by $[\mathscr{F}]$ its class in $K_{0}\left(D^{b}(X)\right)$. Define $\mathcal{Z}([\mathcal{F}])=-\operatorname{deg}(\mathscr{F})+i \operatorname{rk}(\mathscr{F})$ for any $\mathscr{F} \neq 0$. This defines a stability condition and writing $\mathcal{Z}([\mathscr{F}])=r e^{\pi i \theta}$, we find that $\theta=\frac{1}{\pi} \arctan (\operatorname{rk}(\mathscr{F}) /(-\operatorname{deg}(\mathscr{F})))=-\frac{1}{\pi} \arctan (1 /(\mu(\widetilde{F}))$. The subcategory 
$\mathcal{P}(\theta)$ corresponds to the abelian subcategory consisting of semistable bundles of slope $-1 / \tan (\pi \theta)$. It turns out that the set of stability conditions on $D^{b}(X)$ (with some additional finiteness assumptions which we ignore here) admits a natural action of $\widetilde{\mathrm{GL}}_{2}^{+}(\mathbb{R})$, which is free and transitive. For the case of higher genus, we refer to [13].

4.1. Semistable holomorphic bundles of degree 0 . There is an intrinsic definition of semistable bundles over $\mathbb{T}_{\theta}^{\tau}$ (exactly mimicking slope stability) with a notion of Harder-Narasimhan filtration [22]. As observed before, a stability condition on $D^{b}\left(X_{\tau}\right)$ can be constructed by specifying a bounded $t$-structure and group homomorphism from the K-theory of its heart to the complex numbers mapping the effective cone to the upper half plane and having Harder-Narasimhan property. Let us consider the $t$-structure of eqn. (3). It follows from the main result of [20] that the K-theory of the heart of this $t$-structure, which is equivalent to $\operatorname{Vect}\left(\mathbb{T}_{\theta}^{\tau}\right)$, is generated by the classes of the standard bundles $\left[E_{m, n}^{z}\right]$ such that its $\operatorname{rank} \operatorname{rk}\left(E_{m, n}^{z}\right)=m \theta+n$ is positive. In fact, similar to Example 1 we obtain a stability condition on $D^{b}\left(\operatorname{Vect}\left(\mathbb{T}_{\theta}^{\tau}\right)\right) \cong D^{b}\left(X_{\tau}\right)$ given by $\mathcal{Z}\left(\left[E_{m, n}^{z}\right]\right)=-\operatorname{deg}\left(E_{m, n}^{z}\right)+i \operatorname{rk}\left(E_{m, n}^{z}\right)=-m+i(m \theta+n)$. The HarderNarasimhan property follows from the existence of such a filtration for holomorphic bundles (see Proposition 2.8.3 of [22]). This specifies the subcategory of semistable bundles of degree 0 , i.e., $m=0$, which in terms of phase (with respect to this stability condition) is given by $\mathcal{P}(1 / 2)$. Let us denote this category by $\operatorname{SS}_{\mathcal{Z}}\left(0, \mathbb{T}_{\theta}^{\tau}\right)$; it is abelian. Recall that the category $\operatorname{FrVect}\left(\mathbb{\mathbb { T }}_{\theta}^{\tau}\right)$ introduced before Lemma 13 was defined as the extension closed subcategory of $\operatorname{Vect}\left(\mathbb{T}_{\theta}^{\tau}\right)$ generated by the standard modules $E_{1}^{z}$. It follows from Proposition 2.1 part (b) of [23] that the classes [ $\left.E_{1}^{z}\right]$ and $\left[E_{1}^{z^{\prime}}\right]$ are equivalent in $K_{0}\left(\operatorname{Vect}\left(\mathbb{T}_{\theta}^{\tau}\right)\right)$ if and only if $z$ and $z^{\prime}$ denote the same point in $X_{\tau}=\mathbb{C} /(\mathbb{Z}+\tau \mathbb{Z})$. So the classes $\left[E_{1}^{z}\right]$ account for the $\operatorname{Pic}^{0}\left(X_{\tau}\right) \cong X_{\tau}$ part of $K_{0}\left(\operatorname{Vect}\left(\mathbb{T}_{\theta}^{\tau}\right)\right)$ and their extensions account for the full Picard group $\operatorname{Pic}\left(X_{\tau}\right)$.

Lemma 22. The category $\operatorname{FrVect}\left(\mathbb{T}_{\theta}^{\tau}\right)$ is a full subcategory of $\mathrm{SS}_{\mathcal{Z}}\left(0, \mathbb{\mathbb { T }}_{\theta}^{\tau}\right)$.

Proof. Since $E_{1}^{z}$ are indecomposable objects, the Calabi-Yau property of $D^{b}\left(X_{\tau}\right)$ shows that they must be semistable with respect to any stability condition (e.g., Theorem 9.1 of [2]). Moreover, being degree 0 objects with respect to the above stability condition they belong to $S_{\mathcal{Z}}\left(0, \mathbb{T}_{\theta}^{\tau}\right)$. Consequently, the objects obtained by extensions from them are also in $\mathrm{SS}_{\mathcal{Z}}\left(0, \mathbb{T}_{\theta}^{\tau}\right)$, which proves our assertion.

Remark 23. The action of $\widetilde{G L}_{2}^{+}(\mathbb{R})$ on the stability manifold of an elliptic curve is transitive and it simply relabels the phases of semistable objects without altering them. So given any other choice of a stability condition on $D^{b}\left(X_{\tau}\right)$ one may apply an element of $\widetilde{\mathrm{GL}}_{2}^{+}(\mathbb{R})$ to bring it to the form of our chosen stability condition $\mathcal{Z}$.

4.2. Nori finite bundles over $\mathbb{T}_{\boldsymbol{\theta}}^{\boldsymbol{\tau}}$. The aim of this section is to identify the Nori finite bundles over $\mathbb{T}_{\theta}^{\tau}$ and construct a Tannakian category structure on them. 
Let us begin by motivating our choice of $\mathbb{Z}$ as a proposed topological fundamental group of $\mathbb{T}_{\theta}$. It was shown in [15], [4] that noncommutative tori appear at the boundary $\mathbb{P}^{1}(\mathbb{R}) / \operatorname{SL}(2, \mathbb{Z})$ of the moduli space of elliptic curves. At the rational points, which form to a single orbit under $\operatorname{SL}(2, \mathbb{Z})$-action, one has a degenerate elliptic curve, which is topologically equivalent to $\mathbb{C}^{*}$. It is natural to put the degenerate elliptic curves and the noncommutative tori homotopically on the same footing. Let us consider more closely the case of rational $\theta$, say $\theta=p / q$. Then the action of $\mathbb{Z}$ factors through the finite group $\mathbb{Z}_{q}$ and the crossed product $C^{\infty}\left(\mathbb{S}^{1}\right) \rtimes_{p / q} \mathbb{Z}_{q}$ is Morita equivalent to $C^{\infty}\left(\mathbb{S}^{1} / \mathbb{Z}_{q}\right) \simeq C^{\infty}\left(S^{1}\right)$ [26]. We consider the degenerate case with $\theta$ an irrational real number as homotopically equivalent to the finite group case. Therefore, the topological fundamental group of $\mathbb{T}_{\theta}$ is expected to be $\mathbb{Z}$, and we thus look for a Tannakian subcategory of $\operatorname{Vect}\left(\mathbb{T}_{\theta}^{\tau}\right)$ that is equivalent to $\operatorname{Rep}(\mathbb{Z})$.

Theorem 24. The category $\mathcal{B}^{\tau}$ is a full subcategory of $\operatorname{Vect}\left(\mathbb{\nabla}_{\theta}^{\tau}\right)$.

Proof. Since, by Proposition $1, \operatorname{Vect}\left(\mathbb{\nabla}_{\theta}^{\tau}\right)$ and $\operatorname{Vect}\left(\mathbb{T}_{\theta}^{g \tau}\right)$ are equivalent for any $g \in$ $\operatorname{SL}(2, \mathbb{Z})$, we can assume by Lemma 2 that $\tau$ satisfies $\operatorname{wd}(\tau)<1$. As observed in Remark 12, Proposition 8 actually defines a functor from $\mathcal{B}^{\tau}$ to $\operatorname{Vect}\left(\mathbb{\nabla}_{\theta}^{\tau}\right)$. We also denote it by $\psi_{*}$. For $\mathscr{B}^{\tau}$ there is an analogue of Proposition 18: for each object $(M, \nabla)$ in $\mathcal{B}^{\tau}$ there is an isomorphic object $\left(V \otimes \mathcal{O}\left(\mathbb{C}^{*}\right), \delta+A\right)$ with $V$ a vector space and $A$ a constant matrix with all eigenvalues in the same transversal to $\tau \mathbb{Z}$. In fact, $\mathscr{B}^{\tau}$ can be identified with the full subcategory of $\mathscr{B}_{q}^{\tau}$ consisting of those objects with trivial $\mathbb{Z}$ action so that the argument in the proof of Proposition 18 applies directly. The proof of Theorem 19 then implies that $\operatorname{Hom}_{\mathcal{B}^{\tau}}\left(\left(V \otimes \mathcal{O}\left(\mathbb{C}^{*}\right), \delta+A\right),\left(V^{\prime} \otimes \mathcal{O}\left(\mathbb{C}^{*}\right), \delta+A^{\prime}\right)\right)$ can be identified with the space of linear maps $\phi: V \rightarrow V^{\prime}$ that intertwine $A$ and $A^{\prime}$, i.e., so that $\phi A=A^{\prime} \phi$.

Next consider morphisms in the image (under $\psi_{*}$ ) of $\mathcal{B}^{\tau}$ inside $\operatorname{Vect}\left(\mathbb{T}_{\theta}^{\tau}\right)$. The image of the object $\left(V \otimes \mathcal{O}\left(\mathbb{C}^{*}\right), \delta+A\right)$ is clearly $\left(V \otimes \mathcal{A}_{\theta}, \delta_{\tau}+2 \pi i A\right)$ (note the factor of $2 \pi i)$. A morphism between the objects $\left(V \otimes \mathcal{A}_{\theta}, \delta_{\tau}+2 \pi i A\right)$ and $\left(V^{\prime} \otimes \mathcal{A}_{\theta}, \delta_{\tau}+2 \pi i A^{\prime}\right)$ is then given as a map $\phi: V \rightarrow V^{\prime} \otimes A_{\theta}$ that satisfies

$$
\phi \circ\left(\delta_{\tau}+2 \pi i A\right)=\left(\delta_{\tau}+2 \pi i A^{\prime}\right) \circ \phi .
$$

We can decompose $\phi$ as follows: $\phi=\sum_{n_{1}, n_{2} \in \mathbb{Z}} \phi_{n_{1} n_{2}} U_{1}^{n_{1}} U_{2}^{n_{2}}$ and comparing powers of $U_{i}$ on both sides of the previous equation yields

$$
\phi_{n_{1} n_{2}} A=\left(\left(\tau n_{1}+n_{2}\right)+A^{\prime}\right) \phi_{n_{1} n_{2}}
$$

where we factored out a factor of $2 \pi i$ (cf. the definition of $\delta_{\tau}$ ).

As before, this equation implies that $\left(\tau n_{1}+n_{2}\right)+A^{\prime}$ and $A$ have at least one eigenvalue in common. However, this is impossible (unless $n_{1}=n_{2}=0$ ) since the eigenvalues of $A$ and $A^{\prime}$ lie in the same transversal: adding $\tau n_{1}+n_{2}$ will translate every eigenvalue out of the transversal by our assumption $\operatorname{wd}(\tau)<1$. Therefore, 
$\phi_{n_{1} n_{2}}=0$ unless $n_{1}=n_{2}=0$, so that also a morphism in the image of $\mathscr{B}^{\tau}$ in $\operatorname{Vect}\left(\mathbb{\nabla}_{\theta}^{\tau}\right)$ is given by a constant matrix $\phi \in \operatorname{Hom} e\left(V, V^{\prime}\right)$ satisfying $\phi A=A^{\prime} \phi$.

This shows that $\psi_{*}: \mathscr{B}^{\tau} \rightarrow \operatorname{Vect}\left(\mathbb{T}_{\theta}^{\tau}\right)$ is a full embedding. Moreover, the choice of a transversal $\boldsymbol{T}$ to $\tau \mathbb{Z}$ yields a functor from $\operatorname{Rep}(\mathbb{Z})$ to $\mathscr{B}^{\tau}$, which - according to the Riemann-Hilbert correspondence - is an equivalence of tensor categories. We denote this functor again by $F_{\boldsymbol{T}}$ and via the composed functor $\psi_{*} \circ \mathrm{F}_{\boldsymbol{T}}: \operatorname{Rep}(\mathbb{Z}) \rightarrow \operatorname{Vect}\left(\mathbb{T}_{\theta}^{\tau}\right)$ we obtain some holomorphic bundles over $\mathbb{T}_{\theta}^{\tau}$. These bundles are of degree 0 , since the image of $\phi_{*} \circ F_{\boldsymbol{T}}$ is contained in FrVect $\left(\mathbb{T}_{\theta}^{\tau}\right)$ which is a subcategory of $\mathrm{SS}_{\mathcal{Z}}\left(0, \mathbb{T}_{\theta}^{\tau}\right)$. This is our sought-after Tannakian subcategory in $\operatorname{Vect}\left(\mathbb{\mathbb { T }}_{\theta}^{\tau}\right)$, which is equivalent to $\operatorname{Rep}(\mathbb{Z})$.

This leads us to a proposal for the étale fundamental group of the noncommutative torus as follows. Recall that a holomorphic bundle $E$ over a smooth projective variety is called finite if there are two distinct polynomials $f$ and $g$ with non-negative integral coefficients such that $f(V)$ and $g(V)$ are isomorphic. In characteristic zero the Nori finite bundles are equivalent to finite bundles in the above sense. Over a smooth quasiprojective variety a holomorphic vector bundle obtained from a representation of its topological fundamental group that factors through a finite group is Nori finite [17]. The converse is also true, with some additional assumptions, over smooth quasiprojective varieties (see Theorem 3.3 in [1] for the precise statement). For a more elaborate treatment of Nori finite bundles over quasiprojective varieties in characteristic zero we refer the reader to [9]. Motivated by this result we call the objects in the image of $\psi_{*} \circ F_{\boldsymbol{T}}$ which factor through a finite group representation Nori finite over $\mathbb{\mathbb { T }}_{\theta}^{\tau}$ and denote the full subcategory of $\operatorname{Vect}\left(\mathbb{T}_{\theta}^{\tau}\right)$ that they comprise by $\operatorname{NF}\left(\mathbb{T}_{\theta}^{\tau}\right)$. As a result of a theorem proved by Nori in [17], the category $\operatorname{NF}\left(\mathbb{T}_{\theta}^{\tau}\right)$ is Tannakian.

Remark 25. That $\operatorname{NF}\left(\mathbb{T}_{\theta}^{\tau}\right)$ is Tannakian can also be seen directly. Let $f$ be a morphism in $\mathrm{NF}\left(\mathbb{T}_{\theta}^{\tau}\right)$ and, up to isomorphism, we may assume that it is of the form $f:\left(\mathscr{A}_{\theta}^{n_{1}}, \delta_{\tau}+A_{1}\right) \rightarrow\left(\mathscr{A}_{\theta}^{n_{2}}, \delta_{\tau}+A_{2}\right)$, where $A_{i}, i=1,2$, are complex upper triangular matrices of appropriate sizes. The fact that they are in $\operatorname{NF}\left(\mathbb{T}_{\theta}^{\tau}\right)$ means that all the entries are a rational numbers with all eigenvalues in the same transversal. It can be checked that the kernel and the cokernel of $f$ defined in $\operatorname{Vect}\left(\mathbb{T}_{\theta}^{\tau}\right)$ will also have connection matrices with rational entries, which can be brought to the desired form up to isomorphism by suitable base changes. The rigid tensor structure on $\mathfrak{B}^{\tau}$ induces a rigid tensor structure on $\mathrm{NF}\left(\mathbb{T}_{\theta}^{\tau}\right)$ with identity object $\left(\mathscr{A}_{\theta}, \delta_{\tau}\right)$. Moreover, that $\operatorname{End}\left(\mathcal{A}_{\theta}, \delta_{\tau}\right)=\mathbb{C}$ can be seen from the fact that its image in $D^{b}\left(X_{\tau}\right)$ under the Polishchuk-Schwarz equivalence is the stable object $\mathcal{O}_{z=0}$. Finally, the fibre functor on $\mathscr{B}^{\tau}$ restricts to $\operatorname{NF}\left(\mathbb{T}_{\theta}^{\tau}\right)$ turning the latter into a Tannakian category.

Since $\operatorname{NF}\left(\mathbb{T}_{\theta}^{\tau}\right)$ is a subcategory of FrVect $\left(\mathbb{T}_{\theta}^{\tau}\right)$ its image under the functor $\delta_{\tau} \circ \psi_{*}$ lies inside the category of torsion sheaves over $X_{\tau}$. On the other hand the category 
of Nori finite bundles of the complex elliptic curve $X_{\tau}$, denoted by $\mathrm{NF}\left(X_{\tau}\right)$, is also a subcategory of its category of semistable bundles of degree zero. For any $\mu \in \mathbb{Q}$ it is known that the category of semistable bundles of slope $\mu$ over $X_{\tau}$ is equivalent to the category of torsion sheaves over $X_{\tau}$ by some Fourier-Mukai transform. In this specific case the category of semistable bundles of degree 0 (hence slope 0 ) can be made equivalent to the category of torsion sheaves by applying a specific SeidelThomas twist functor (for the details see [30]). The effect of this functor on $(\mathrm{deg} \mathrm{rk})^{t}$, viewed as a column vector, is simply left multiplication by the matrix $\left(\begin{array}{cc}0 & 1 \\ -1 & 0\end{array}\right)$, i.e, degree 0 bundles become rank 0 torsion sheaves. Therefore, $\operatorname{NF}\left(\mathbb{T}_{\theta}^{\tau}\right)$ and $\operatorname{NF}\left(X_{\tau}\right)$ can both be viewed as subcategories of the category of torsion sheaves of $X_{\tau}$. The set of complex points of the Tannakian group of $\operatorname{NF}\left(X_{\tau}\right)$ is isomorphic to $\hat{\mathbb{Z}} \times \hat{\mathbb{Z}}$, while that of $\operatorname{NF}\left(\mathbb{\mathbb { T }}_{\theta}^{\tau}\right)$ is simply $\widehat{\mathbb{Z}}$. This is not very surprising since over an algebraically closed field of characteristic zero, Nori's fundamental group scheme is the same as Grothendieck's étale fundamental group (ignoring the difference that the former is a pro-group scheme and the latter is only an abstract group). The pinching of one of the homology cycles at the rational boundary of the modular curve (and possibly its deformation for noncommutative tori) accounts for the lack of one copy of $\hat{\mathbb{Z}}$ in the étale fundamental group of $\mathbb{T}_{\theta}^{\tau}$.

\section{References}

[1] I. Biswas, Y. I. Holla, and G. Schumacher, On a characterization of finite vector bundles as vector bundles admitting a flat connection with finite monodromy group. Proc. Amer. Math. Soc. 128 (2000), 3661-3669. Zbl 0977.53023 MR 1695096

[2] T. Bridgeland, Stability conditions on triangulated categories. Ann. of Math. (2) 166 (2007), 317-345. Zbl 1137.18008 MR 2373143

[3] A. Connes, $C^{*}$ algèbres et géométrie différentielle. C. R. Acad. Sci. Paris Sér. A 290 (1980), 599-604. Zbl 0433.46057 MR 572645

[4] A. Connes, M. R. Douglas, and A. Schwarz, Noncommutative geometry and matrix theory: compactification on tori. J. High Energy Phys. 02 (1998), 003. Zbl 1018.81052 MR 1613978

[5] A. Connes and M. A. Rieffel, Yang-Mills for non-commutative two-tori. In Operator algebras and mathematical physics (Iowa City, Iowa, 1985), Contemp. Math. 62, Amer. Math. Soc., Providence, RI, 1987, 237-266. Zbl 0633.46069 MR 0878383

[6] P. Deligne, Équations différentielles à points singuliers réguliers. Lecture Notes in Math. 163, Springer-Verlag, Berlin 1970. Zbl 0244.14004 MR 0417174

[7] P. Deligne, Catégories tannakiennes. In The Grothendieck Festschrift, Vol. II, Progr. Math. 87, Birkhäuser, Boston 1990, 111-195. Zbl 0727.14010 MR 1106898

[8] P. Deligne and J. Milne, Tannakian categories. In Hodge cycles, motives and Shimura varieties, Lecture Notes in Math. 900, Springer-Verlag, Berlin 1982, 101-228. Zbl 0477.14004 MR 0654325 
[9] H. Esnault and P. H. Hai, The fundamental groupoid scheme and applications. Ann. Inst. Fourier (Grenoble) 58 (2008), 2381-2412. Zbl 05505487

[10] L. Fuchs and L. Salce, Modules over valuation domains. Lecture Notes Pure Appl. Math. 97, Marcel Dekker, New York 1985. Zbl 0578.13004 MR 0786121

[11] B. Keller, Deriving DG categories. Ann. Sci. École Norm. Sup. (4) 27 (1994), 63-102. Zbl 0799.18007 MR 1258406

[12] M. Kontsevich, XI Solomon Lefschetz Memorial Lecture Series: Hodge structures in non-commutative geometry. In Non-commutative geometry in mathematics and physics (Mexico City, 2005), Contemp. Math. 462, Amer. Math. Soc., Providence, RI, 2008, 1-21. Zbl 05343346 MR 2444365

[13] E. Macrì, Stability conditions on curves. Math. Res. Lett. 14 (2007), 657-672. Zbl 1151.14015 MR 2335991

[14] B. Malgrange, Regular connections, after Deligne. In Algebraic D-modules. Perspect. Math. 2, Academic Press, Boston 1987, 151-172. Zbl 0642.32001 MR 0882000

[15] Yu. I. Manin and M. Marcolli, Continued fractions, modular symbols, and noncommutative geometry. Selecta Math. (N.S.) 8 (2002), 475-521. Zbl 1116.11033 MR 1931172

[16] M. V. Nori, On the representations of the fundamental group. Compositio Math. 33 (1976), 29-41. Zbl 0337.14016 MR 0417179

[17] M. V. Nori, The fundamental group-scheme. Proc. Indian Acad. Sci. Math. Sci. 91 (1982), 73-122. Zbl 0586.14006 MR 682517

[18] M. Pimsner and D. Voiculescu, Imbedding the irrational rotation $C^{*}$-algebra into an AF-algebra. J. Operator Theory 4 (1980), 201-210. Zbl 0525.46031 MR 595412

[19] A. Y. Pirkovskii, On certain homological properties of Stein algebras. J. Math. Sci. 95 (1999), 2690-2702. Zbl 0944.46048 MR 1712995

[20] A. Polishchuk, Classification of holomorphic vector bundles on noncommutative two-tori. Doc. Math. 9 (2004), 163-181. Zbl 1048.32012 MR 2054986

[21] A. Polishchuk, Noncommutative two-tori with real multiplication as noncommutative projective varieties. J. Geom. Phys. 50 (2004), 162-187. Zbl 1085.14003 MR 2078224

[22] A. Polishchuk, Quasicoherent sheaves on complex noncommutative two-tori. Selecta Math. (N.S.) 13 (2007), 137-173. Zbl 1135.32011 MR 2330589

[23] A. Polishchuk and A. Schwarz, Categories of holomorphic vector bundles on noncommutative two-tori. Comm. Math. Phys. 236 (2003), 135-159. Zbl 1033.58009 MR 1977884

[24] M. van der Put, Differential Galois theory, universal rings and universal groups. In Differential algebra and related topics, World Scientific Publ., Singapore 2002, 171-189. Zbl 1070.12003 MR 1921699

[25] M. van der Put and M. F. Singer, Galois theory of linear differential equations. Grundlehren Math. Wiss. 328, Springer-Verlag, Berlin 2003. Zbl 1036.12008 MR 1960772

[26] M. A. Rieffel, Morita equivalence for $C^{*}$-algebras and $W^{*}$-algebras. J. Pure Appl. Algebra 5 (1974), 51-96. Zbl 0295.46099 MR 0367670 
[27] M. A. Rieffel, $C^{*}$-algebras associated with irrational rotations. Pacific J. Math. 93 (1981), 415-429. Zbl 0499.46039 MR 623572

[28] M. A. Rieffel, Non-commutative tori - a case study of non-commutative differentiable manifolds. In Geometric and topological invariants of elliptic operators, Contemp. Math. 105, Amer. Math. Soc., Providence, RI, 1990, 191-211. Zbl 0713.46046 MR 1047281

[29] N. Saavedra Rivano, Catégories Tannakiennes. Lecture Notes in Math. 265, SpringerVerlag, Berlin 1972. Zbl 0241.14008 MR 0338002

[30] P. Seidel and R. Thomas, Braid group actions on derived categories of coherent sheaves. Duke Math. J. 108 (2001), 37-108. Zbl 1092.14025 MR 1831820

[31] Y. Soibelman and V. Vologodsky, Noncommutative compactifications and elliptic curves. Internat. Math. Res. Notices 2003 (2003), 1549-1569. Zbl 1052.14004 MR 1976601

[32] W. Wasow, Asymptotic expansions for ordinary differential equations. Robert E. Krieger Publishing Co., Huntington, N.Y., 1976. Zbl 0644.34003 MR 0460820

Received April 21, 2008; revised September 11, 2008

S. Mahanta, Department of Mathematics, University of Toronto, 40 St George Street, Toronto, ON M5S 2E4, Canada, and Institut des Hautes Études Scientifiques,

Le Bois-Marie 35, route de Chartres, 91440 Bures-sur-Yvette, France

E-mail: smahanta@ihes.fr

W. D. van Suijlekom, Institute for Mathematics, Astrophysics and Particle Physics, Faculty of Science, Radboud Universiteit Nijmegen, Toernooiveld 1, 6525 ED Nijmegen,

The Netherlands

E-mail: waltervs@math.ru.nl 